\title{
Massive QTL analysis identifies pleiotropic genetic determinants for stress resistance, aroma formation, and ethanol, glycerol and isobutanol production in Saccharomyces cerevisiae
}

Ping-Wei Ho ${ }^{1,2,3}$, Supinya Piampongsant ${ }^{1,2,3}$, Brigida Gallone ${ }^{1,2,3}$, Andrea Del Cortona ${ }^{1,2,3}$, Pieter-Jan Peeters ${ }^{1,2,3}$, Frank Reijbroek ${ }^{1,2,3}$, Jules Verbaet ${ }^{1,2,3}$, Beatriz Herrera ${ }^{1,2,3}$, Jeroen Cortebeeck ${ }^{1,2,3}$, Robbe Nolmans $s^{1,2,3}$, Veerle Saels ${ }^{1,2,3}$, Jan Steensels ${ }^{1,2,3}$, Daniel F. Jarosz $z^{4,5}$ and Kevin J. Verstrepen ${ }^{1,2,3,6^{*}}$ (D)

\begin{abstract}
Background: The brewer's yeast Saccharomyces cerevisiae is exploited in several industrial processes, ranging from food and beverage fermentation to the production of biofuels, pharmaceuticals and complex chemicals. The large genetic and phenotypic diversity within this species offers a formidable natural resource to obtain superior strains, hybrids, and variants. However, most industrially relevant traits in S. cerevisiae strains are controlled by multiple genetic loci. Over the past years, several studies have identified some of these QTLs. However, because these studies only focus on a limited set of traits and often use different techniques and starting strains, a global view of industrially relevant QTLs is still missing.

Results: Here, we combined the power of 1125 fully sequenced inbred segregants with high-throughput phenotyping methods to identify as many as 678 QTLs across 18 different traits relevant to industrial fermentation processes, including production of ethanol, glycerol, isobutanol, acetic acid, sulfur dioxide, flavor-active esters, as well as resistance to ethanol, acetic acid, sulfite and high osmolarity. We identified and confirmed several variants that are associated with multiple different traits, indicating that many QTLs are pleiotropic. Moreover, we show that both rare and common variants, as well as variants located in coding and non-coding regions all contribute to the phenotypic variation.

Conclusions: Our findings represent an important step in our understanding of the genetic underpinnings of industrially relevant yeast traits and open new routes to study complex genetics and genetic interactions as well as to engineer novel, superior industrial yeasts. Moreover, the major role of rare variants suggests that there is a plethora of different combinations of mutations that can be explored in genome editing.
\end{abstract}

*Correspondence: kevin.verstrepen@kuleuven.be

${ }^{6}$ Labo VIB-CMPG, Bio-Incubator, Gaston Geenslaan 1, 3001 Leuven, Heverlee, Belgium

Full list of author information is available at the end of the article

\section{Background}

The brewer's yeast Saccharomyces cerevisiae plays a key role in the production of fermented foods, beverages, biofuels and pharmaceuticals. Despite S. cerevisiae being generally suited for industrial applications, there is an original author(s) and the source, provide a link to the Creative Commons licence, and indicate if changes were made. The images or other third party material in this article are included in the article's Creative Commons licence, unless indicated otherwise in a credit line to the material. If material is not included in the article's Creative Commons licence and your intended use is not permitted by statutory regulation or exceeds the permitted use, you will need to obtain permission directly from the copyright holder. To view a copy of this licence, visit http://creativecommons.org/licenses/by/4.0/. The Creative Commons Public Domain Dedication waiver (http://creativeco mmons.org/publicdomain/zero/1.0/) applies to the data made available in this article, unless otherwise stated in a credit line to the data. 
enormous genetic and phenotypic diversity among different $S$. cerevisiae strains, with strains typically excelling in some areas, but scoring worse for other important phenotypes [1]. Moreover, the strains currently used for industrial production only represent a small fraction of the natural diversity and do not always perform optimally [1-6]. Hence, one obvious yet effective path towards superior industrial yeasts with improved fermentation properties is through the exploration and exploitation of the natural yeast biodiversity $[7,8]$. Aside from selecting natural strains that boast a desirable combination of phenotypes, it is also possible to cross multiple strains and select hybrids that combine and improve upon specific traits [7-9]. However, although this approach has proven extremely powerful, it also suffers from important shortcomings. Firstly, natural yeast strains rarely combine all desired traits. Secondly, while hybrids allow combining useful traits, the very process of shuffling genomes is relatively slow and labor-intensive, and inevitably also leads to loss of some positive properties.

An alternative route to exploit the natural diversity of yeasts is to employ genetic engineering to combine specific mutations and alleles that contribute to desirable phenotypes. The use of various CRISPR-based techniques has now made it relatively easy to introduce a series of desirable allele combinations into an existing yeast, to further improve one or several phenotypes while not affecting the rest of the genome and thus maximizing the conservation of other traits [10-12].

Despite the promise of genetic modification and genome editing to obtain superior industrial microbes, identification of the genetic determinants (gene alleles and other genetic variations) that are responsible for desired traits remains a major challenge. Many industrially relevant phenotypes are quantitative, involving multiple genetic loci (called quantitative trait loci, QTLs) that can sometimes show complex genetic interactions [13]. One frequently used approach to dissect QTLs is experimental evolution and re-sequencing (E\&R). By monitoring the genetic changes between isogenic populations as they adapt to specific selective pressure across multiple generations, the causative loci underlying the selected trait may be identified [14-16]. Using this approach, studies have for example pinpointed mutations that contribute to ethanol tolerance [17], glycerol utilization [18], and heat resistance [19]. However, although E\&R can serve as a powerful tool, it often leads to physiological trade-offs between a selected trait and other aspects of yeast. Additionally, an E\&R approach can only be exploited for evolutionarily selectable phenotypes, such as improvements in growth rate, tolerance, or substrate utilization. However, if the desired phenotype is not inherently selectable or counter-productive for cell's fitness (e.g., overproduction of specific metabolites), it prevents straightforward selection through evolution.

Another approach used to link a complex trait to its genetic underpinning is quantitative trait loci (QTL) mapping [20-23]. QTL mapping takes advantage of $S$. cerevisiae's meiotic recombination efficiency to determine the extent of co-segregation between loci with known positions (genetic variations) and the genetic determinants of the phenotype of interest, whose positions are unknown $[24,25]$. This method has been successfully applied to reveal the molecular basis of several quantitative traits such as heat tolerance [26], acetic acid tolerance $[27,28]$, ethanol tolerance $[29,30]$ or wine aroma [31].

Despite the ever-increasing list of identified QTLs in yeast and other organisms, QTL mapping generally suffers from low throughput due to the large body of work required to map and confirm one locus for one specific property [32,33]. Additionally, different studies mostly focus on one phenotype at a time. Hence, it is mostly unclear whether the same QTL might influence multiple phenotypes (positively or negatively). Moreover, because most studies do not map identified QTLs onto the S. cerevisiae phylogeny, the evolutionary history and distribution of QTLs across the S. cerevisiae population is also still largely unknown.

Here, we employ the approach first described by She \& Jarosz in 2018 that enables systematic identification of QTLs across multiple traits. This method takes advantage of the decreased sequencing cost and the possibilities of high-throughput phenotyping for yeast to map multiple traits in one large-scale mapping effort. Specifically, the approach exploits a large-scale inbreeding crossing scheme and in silico fine-mapping to identify causative variants at single-nucleotide resolution, while maintaining high statistical power. Using this setup, causal variants have been pinpointed across a wide array of ecologically relevant traits, including drug resistance, carbon source utilization, and chemical stressors [34-36]. Here, we combined the power of the 1125 previously sequenced inbred segregants with large-scale phenotyping methods to pinpoint 678 QTLs that together determine 18 different yeast traits relevant to industrial fermentations and biotechnological processes. From this set, we identified several key variants that influence the production of fermentative metabolites, including ethanol, glycerol, isobutanol, acetic acid, and sulfur dioxide, as well as resistance to ethanol, acetic acid, sulfite, and high osmolarity and salinity. The large number of QTLs identified reflects the genetic complexity of the traits we examined. Thanks to the large segregant population, we were able to resolve 254 QTLs to single-nucleotide level (QTN) and an additional 58 to single gene (size of about 
$1 \mathrm{~kb})$. Interestingly, we show that pleiotropy plays a major role in phenotypic diversity of industrially relevant yeast characteristics. For example, a (previously introduced) auxotrophic marker in one of the parental strains affected 12 traits, while a specific rearrangement in the subtelomeric region of chromosome VII affected at least 5 phenotypes. Lastly, we show that several of the causative mutations exhibit similar phenotypic effects when introduced to other, genetically distinct industrial strains. This not only confirms the accuracy of our approach, but also opens new routes to study complex genetics and genetic interactions as well as to engineer novel, superior industrial yeasts.

\section{Results}

The main aim of this study was to obtain a comprehensive view of the different QTLs that contribute to industrially relevant properties in S. cerevisiae. To this end, $1125 \mathrm{~F}_{6}$ segregants were obtained from a cross of two phenotypically divergent $S$. cerevisiae strains, RM11-1a, a natural vineyard isolate, and YJM975 $\alpha$, originally isolated from an immunocompromised patient in Italy [34]. We reasoned that this combination of one industrial, and one non-industrial strain would maximize our chances of identifying QTLs that are specific for strains adapted to industrial conditions, while possibly also revealing to what extent non-industrial strains also harbor other industrially relevant alleles. For each of the 1125 segregants and the two parental yeasts, we set up small-scale fermentation reactions in medium and conditions mimicking industrial beer production. For each of these 1127 fermentations, we measured 18 different industrially relevant parameters, including the production of primary and secondary metabolites. In addition, we screened all 1125 segregants for their resistance to various industrially relevant stress factors and consumption of maltose. This large set of phenotypic data was subsequently combined with the available genome sequences of each of the segregants and analyzed using the pipeline developed by She and Jarosz (2018) to identify QTLs underlying the phenotypes. Finally, some of the QTLs were experimentally confirmed to verify the mapping and explore the possibility of using the data to engineer superior industrial yeasts.

\section{Identification of $L E U 2$ as a major pleiotropic QTL}

Most traits show a normal distribution within the population, a typical characteristic of complex phenotypes that are controlled by multiple different genetic loci (Fig. 1). For a few phenotypes, however, the $F_{6}$ progeny shows a clear bimodal segregation, indicative of one major segregating QTL that has substantial influence.
One such QTL was the LEU2 marker, a gene encoding beta-isopropylmalate dehydrogenase that catalyzes the third step in leucine biosynthesis which was deleted in RM11-1a to facilitate selection of progeny during the repeated rounds of crossing [34]. The absence or presence of LEU2 drastically influences the progeny's phenotypes, especially in the production of metabolites that are directly related to amino acid and nucleic acid metabolism: isoamyl alcohol, isoamyl acetate, isobutanol, 1-propanol, ethyl octanoate, ethyl hexanoate, acetic acid, and $\mathrm{SO}_{2}$ (Additional file 2: Table S1). Hence, the marker functions as an artificial QTL and serves as a positive control for the QTL pipeline. Perhaps unsurprisingly, the most prominent effect of the absence or presence of $L E U 2$ is the formation of isoamyl alcohol, which is directly related to the leucine biosynthetic pathway in which Leu2 is involved. Segregants that harbor LEU2 produce 193\% more isoamyl alcohol in average compared to strains lacking it $(p<0.001)$ (Additional file 1: Figure S1). Because the effect of $L E U 2$ on many phenotypes is particularly strong and partly obscures more subtle differences related to natural QTLs, the segregants lacking LEU 2 were omitted and only the 845 prototrophic $\mathrm{F}_{6}$ segregants were used for the subsequent QTL analysis.

\section{Variance in industrial traits is driven by both rare and common, coding and intergenic variants}

Analysis of 18 different industrially relevant phenotypes of the prototrophic $\mathrm{F}_{6}$ segregants yielded a total of 678 QTLs with $p<10^{-5}$ (Additional file 2: Table S2). Of the QTLs identified, 21 are small insertion or deletions (Indels) and 657 are single-nucleotide polymorphisms (SNPs). 254 QTLs could be unambiguously mapped to single-nucleotide resolution (QTN), and 58 could be mapped to single gene-level (size about $1 \mathrm{~Kb}$ ). The QTNs responsible for these diverse traits included missense and intergenic (non-coding) variants, as well as synonymous and missense variants in coding regions (Fig. 2A). Roughly $43.7 \%$ of the QTNs locate in intergenic regions $(N=111)$, while synonymous and missense mutations each account for $23.2 \%(N=59)$ and $29.1 \%(N=74)$ of the QTNs, respectively. This distribution is very similar to the distribution of all variants between the founder strains (i.e., all polymorphisms between the two parental strains, irrespective of whether they are linked to a QTN or not; Fig. 2B). The presence of a large fraction of non-coding QTNs, including regulatory variants, is in line with the observation of former studies employing the same cross (Fig. 2B), where the complex traits tested were also fueled by polymorphisms of different molecular classes [34, 36].

As the majority of the industrially relevant traits included in the current study relate to fermentation 


\begin{tabular}{|c|c|c|c|c|c|c|c|c|c|c|c|c|c|c|c|c|c|c|}
\hline $\begin{array}{l}\text { 1-propanol } \\
\text { conc. }\end{array}$ & & $\begin{array}{c}\text { rho: } \\
\text { o. } 44^{* * *}\end{array}$ & $\begin{array}{l}\text { rho: } \\
0.44^{* \ldots *}\end{array}$ & $\begin{array}{l}\text { rho: } \\
0.30 * \ldots\end{array}$ & $\begin{array}{c}\text { rho: } \\
0.50^{*+*}\end{array}$ & $\begin{array}{c}\text { hho: } \\
0.18^{\mathrm{m}}\end{array}$ & $\begin{array}{l}\text { rho: } \\
\text { r. } 37 \times+\ldots\end{array}$ & $\begin{array}{l}\text { rho: } \\
-0.04\end{array}$ & $\begin{array}{l}\text { rho: } \\
-0.03\end{array}$ & $\begin{array}{l}\text { rho: } \\
-0.05\end{array}$ & $\begin{array}{l}\text { rho: } \\
0.18 * \ldots\end{array}$ & $\begin{array}{l}\text { rho: } \\
-0.03\end{array}$ & $\begin{array}{l}\text { rho: } \\
0.15^{\mathrm{m}}\end{array}$ & $\begin{array}{l}\text { rho: } \\
\text { o. } 16^{\mathrm{*a*}}\end{array}$ & $\begin{array}{l}\text { rho: } \\
0.34 * \ldots\end{array}$ & $\begin{array}{c}\text { rho: } \\
-0.11+\ldots\end{array}$ & $\begin{array}{c}\text { rho: } \\
0.26^{\mathrm{kn*}}\end{array}$ & $\begin{array}{l}\text { rho: } \\
-0.08 *\end{array}$ \\
\hline $\begin{array}{l}\text { ethyl acetate } \\
\text { conc. }\end{array}$ & $\%$ & & $\begin{array}{c}\text { rho: } \\
0.37 \ldots *\end{array}$ & $\begin{array}{l}\text { rho: } \\
0.32 * *+\end{array}$ & $\begin{array}{c}\text { rho: } \\
0.14^{* * *}\end{array}$ & $\begin{array}{c}\text { hho: } \\
\text { o. } 15^{\mathrm{*} *}\end{array}$ & $\begin{array}{l}\text { rho: } \\
\text { o.41*** }\end{array}$ & $\begin{array}{l}\text { rho: } \\
0.21^{* \ldots+}\end{array}$ & $\begin{array}{l}\text { rho: } \\
-0.06 .\end{array}$ & $\begin{array}{l}\text { rho: } \\
-0.02\end{array}$ & $\begin{array}{l}\text { rho: } \\
-0.11^{* *}\end{array}$ & $\begin{array}{l}\text { rho: } \\
-0.01\end{array}$ & $\begin{array}{l}\text { rho: } \\
-0.03\end{array}$ & $\begin{array}{c}\text { rho: } \\
-0.11^{* \star *}\end{array}$ & $\begin{array}{l}\text { rho: } \\
0.37 \times+\ldots\end{array}$ & $\begin{array}{l}\text { rho: } \\
0.12 * \star\end{array}$ & $\begin{array}{l}\text { rho: } \\
0.07 *\end{array}$ & $\begin{array}{l}\text { rho: } \\
0.11 * *\end{array}$ \\
\hline $\begin{array}{l}\text { ethyl hexanoate } \\
\text { conc. }\end{array}$ & 6 & 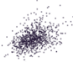 & & $\begin{array}{c}\text { hho: } \\
0.72^{*+*}\end{array}$ & $\begin{array}{l}\text { rho: } \\
\text { o. } 52^{* * *}\end{array}$ & $\begin{array}{l}\text { rho: } \\
-0.05 .\end{array}$ & $\begin{array}{l}\text { rho: } \\
0.64 * \ldots+\end{array}$ & $\begin{array}{c}\text { xho: } \\
0.17 * * \star\end{array}$ & $\begin{array}{c}\text { rho: } \\
-0.12 * * *\end{array}$ & $\begin{array}{l}\text { rho: } \\
0.07 *\end{array}$ & $\begin{array}{l}\text { rho: } \\
0.26 * * *\end{array}$ & $\begin{array}{l}\text { rho: } \\
0.03\end{array}$ & $\begin{array}{l}\text { rho: } \\
0.17^{*+*}\end{array}$ & $\begin{array}{l}\text { rho: } \\
0.07 *\end{array}$ & $\begin{array}{c}\text { rho: } \\
0.11+* \star\end{array}$ & $\begin{array}{c}\text { rho: } \\
-0,34+2 *\end{array}$ & $\begin{array}{l}\text { rho: } \\
0.07 *\end{array}$ & $\begin{array}{c}\text { rhos: } \\
-0.10 * *\end{array}$ \\
\hline $\begin{array}{l}\text { ethyl octanoate } \\
\text { conc. }\end{array}$ & ) & \% & ) & & $\begin{array}{c}\text { rho: } \\
0.38 * \ldots\end{array}$ & $\begin{array}{c}\text { rho: } \\
-0.16 * t+\end{array}$ & $\begin{array}{c}\text { rho: } \\
0.57 \times+*\end{array}$ & $\begin{array}{l}\text { rho: } \\
0.18^{* * *}\end{array}$ & $\begin{array}{c}\text { rho: } \\
-0.14^{* * *}\end{array}$ & $\begin{array}{l}\text { rho: } \\
\text { o. }\end{array}$ & $\begin{array}{c}\text { rho: } \\
0.28^{* * *}\end{array}$ & $\begin{array}{l}\text { rho: } \\
\text { o. } 0.08 *\end{array}$ & $\begin{array}{c}\text { rho: } \\
0.20 * * *\end{array}$ & $\begin{array}{c}\text { rho: } \\
-0.06 .\end{array}$ & $\begin{array}{l}\text { rho: } \\
0.10^{* *}\end{array}$ & $\begin{array}{l}\text { rho: } \\
-0.30 * * *\end{array}$ & $\begin{array}{l}\text { rho: } \\
-0.04\end{array}$ & $\begin{array}{c}\text { rho: } \\
-0.00 * *\end{array}$ \\
\hline $\begin{array}{l}\text { isoamyl alcohol } \\
\text { conc. }\end{array}$ & ) & 8 & ) & 4 & the & $\begin{array}{l}\text { rho: } \\
0.09 *+\end{array}$ & $\begin{array}{l}\text { rho: } \\
0.68 \times+\ldots\end{array}$ & $\begin{array}{l}\text { rho: } \\
0.07 *\end{array}$ & $\begin{array}{c}\text { rho } \\
-0.20^{\circ+*}\end{array}$ & $\begin{array}{l}\text { rho: } \\
0.04\end{array}$ & $\begin{array}{c}\text { rho: } \\
0.43 * \ldots\end{array}$ & $\begin{array}{c}\text { rho: } \\
0.12 * *\end{array}$ & $\begin{array}{c}\text { hho: } \\
0.22^{* * *}\end{array}$ & $\begin{array}{c}\text { rho: } \\
0.13 * * *\end{array}$ & $\begin{array}{c}\text { rho: } \\
0.15 * *\end{array}$ & $\begin{array}{c}\text { rho: } \\
-0.47 * \ldots+\end{array}$ & $\begin{array}{l}\text { rho: } \\
0.17+\ldots+\end{array}$ & $\begin{array}{c}\text { rho: } \\
-0.32+\ldots+\end{array}$ \\
\hline $\begin{array}{l}\text { isobutanol } \\
\text { conc. }\end{array}$ & y & 36 & (1) & 4 & (1) & & $\begin{array}{l}\text { rho: } \\
0.01\end{array}$ & $\begin{array}{c}\text { rho: } \\
0.16^{* * *}\end{array}$ & $\begin{array}{c}\text { rho: } \\
-0.10 * *\end{array}$ & $\begin{array}{l}\text { rho: } \\
0.05\end{array}$ & $\begin{array}{l}\text { rho: } \\
-0.19 * * *\end{array}$ & $\begin{array}{l}\text { rho: } \\
-0.00\end{array}$ & $\underset{\substack{\text { rho: } \\
-0.11 * * *}}{\mid}$ & $\begin{array}{l}\text { rho: } \\
0.29 * * *\end{array}$ & $\begin{array}{l}\text { rho: } \\
-0.05\end{array}$ & $\begin{array}{l}\text { rho: } \\
0.05\end{array}$ & $\begin{array}{l}\text { rho: } \\
0.34 * * *\end{array}$ & $\begin{array}{l}\text { rho: } \\
0.25 * * *\end{array}$ \\
\hline $\begin{array}{l}\text { isopentyl acetate } \\
\text { conc. }\end{array}$ & 4 & 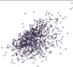 & 6 & 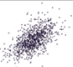 & \% & 1 & & $\begin{array}{c}\text { rho: } \\
0.48 * \ldots *\end{array}$ & $\begin{array}{c}\text { rho: } \\
-0.21+* *\end{array}$ & $\begin{array}{c}\text { rho: } \\
0.12 * *\end{array}$ & $\begin{array}{l}\text { rho: } \\
0.34 * * *\end{array}$ & $\begin{array}{c}\text { rho: } \\
\text { o. } 114 * *\end{array}$ & $\begin{array}{l}\text { rho: } \\
0.19 * *\end{array}$ & $\begin{array}{c}\text { rho: } \\
0.16 * * *\end{array}$ & 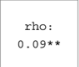 & $\begin{array}{c}\text { rho: } \\
-0,37 * * *\end{array}$ & $\begin{array}{l}\text { rho: } \\
0.11+\ldots\end{array}$ & $\begin{array}{l}\text { rho: } \\
-0.19 \mathrm{w*}\end{array}$ \\
\hline $\begin{array}{r}\text { phenethyl acetate } \\
\text { conc. }\end{array}$ & 4 & r & \% & ? & W & X & 8 & & $\begin{array}{c}\text { rho: } \\
-0.12^{2 * *}\end{array}$ & $\begin{array}{l}\text { rho: } \\
0.14 * \ldots\end{array}$ & $\begin{array}{l}\text { rho: } \\
-0.06 .\end{array}$ & $\begin{array}{c}\text { rho: } \\
\text { o.10** }\end{array}$ & $\begin{array}{l}\text { rho: } \\
-0.01\end{array}$ & $\begin{array}{l}\text { rho: } \\
0.32+* *\end{array}$ & $\begin{array}{l}\text { rho: } \\
-0.06 .\end{array}$ & $\begin{array}{c}\text { rho: } \\
-0.06 .\end{array}$ & $\begin{array}{l}\text { rho: } \\
0.28+\ldots *\end{array}$ & $\begin{array}{c}\text { rhos: } \\
0.24 * * *\end{array}$ \\
\hline osmotolerance & W & (x) & 4 & 14 & r. & W & 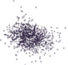 & 4 & & $\begin{array}{c}\text { rho: } \\
-0.12^{2 * * *}\end{array}$ & $\begin{array}{l}\text { rho: } \\
0.05\end{array}$ & $\begin{array}{c}\text { rho: } \\
-0.17 * * *\end{array}$ & $\begin{array}{l}\text { rho: } \\
0.02\end{array}$ & $\begin{array}{c}\text { rho: } \\
-0.15 * * *\end{array}$ & $\begin{array}{c}\text { rho: } \\
-0.12 * * *\end{array}$ & $\begin{array}{l}\text { rho: } \\
\text { r.t15*** }\end{array}$ & $\begin{array}{l}\text { rho: } \\
-0.04\end{array}$ & $\begin{array}{l}\text { rho: } \\
-0.00\end{array}$ \\
\hline $\begin{array}{r}\mathrm{SO} 3 \\
\text { tolerance }\end{array}$ & 4 & 4 & \% & 6 & 6 & W & N & \% & \% & & $\begin{array}{l}\text { rho: } \\
0.04\end{array}$ & $\begin{array}{l}\text { rho: } \\
-0.04\end{array}$ & $\begin{array}{c}\text { rho: } \\
0.20 * * *\end{array}$ & $\begin{array}{l}\text { rho: } \\
0.09 * *\end{array}$ & $\begin{array}{l}\text { rho: } \\
-0.00\end{array}$ & $\begin{array}{c}\text { rho: } \\
-0.09 * *\end{array}$ & $\begin{array}{l}\text { rho: } \\
0.06 .\end{array}$ & $\begin{array}{l}\text { rho: } \\
0.04\end{array}$ \\
\hline $\begin{array}{c}\text { acetic acid } \\
\text { tolerance }\end{array}$ & \% & 4 & \% & \% & W & 1) & \% & 8 & 1) & 6 & & $\begin{array}{l}\text { rho: } \\
-0.04\end{array}$ & $\begin{array}{l}\text { rho: } \\
0.26 * * x\end{array}$ & $\begin{array}{l}\text { rho: } \\
-0.03\end{array}$ & $\begin{array}{l}\text { rho: } \\
-0.03\end{array}$ & $\begin{array}{c}\text { rho: } \\
-0.34 * * *\end{array}$ & $\begin{array}{c}\text { sho: } \\
-0.04\end{array}$ & $\begin{array}{c}\text { rho: } \\
-0.31+* *\end{array}$ \\
\hline $\begin{array}{c}\text { ethanol } \\
\text { tolerance }\end{array}$ & 6) & W & 4 & 4 & 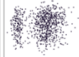 & (a) & X & \% & X & 4 & (4) & & $\begin{array}{l}\text { rho: } \\
\text { o. } 06 .\end{array}$ & $\begin{array}{c}\text { rho: } \\
0.12 * * x\end{array}$ & $\begin{array}{l}\text { rho: } \\
-0.03\end{array}$ & $\begin{array}{l}\text { rho: } \\
-0.08 x\end{array}$ & $\begin{array}{l}\text { rho: } \\
0.03\end{array}$ & $\begin{array}{l}\text { rho: } \\
0.01\end{array}$ \\
\hline halotolerance & ( & W & 4 & (6) & 16 & 4 & 6 & 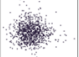 & N: & 4 & 4 & 3. & & $\begin{array}{l}\text { fho: } \\
-0.04\end{array}$ & $\begin{array}{l}\text { rho: } \\
0.01\end{array}$ & $\begin{array}{c}\text { rho: } \\
-0.22 * * *\end{array}$ & $\begin{array}{l}\text { hho: } \\
\text { o. o3 }\end{array}$ & $\begin{array}{c}\text { rho: } \\
-0.10 * *\end{array}$ \\
\hline maltose growth & 3 & r & 18 & 6x & ( & 秝 & ty & (4) & 4 & ) & X & r) & ) & the & $\begin{array}{l}\text { rho: } \\
0.11 * * *\end{array}$ & $\begin{array}{l}\text { rho: } \\
0.04 \\
\text { hou }\end{array}$ & $\begin{array}{l}\text { rho: } \\
0.44 * x *\end{array}$ & $\begin{array}{c}\text { rho: } \\
0.11 * * *\end{array}$ \\
\hline $\begin{array}{c}\text { ethanol } \\
\text { conc. }\end{array}$ & 5 & 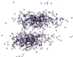 & $=$ & \% & ? & te. & s. & $=$ & 6 & - & (4) & (4): & 4 & 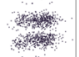 & & $\begin{array}{c}\text { rho: } \\
0.14 * * *\end{array}$ & $\begin{array}{c}\text { rho: } \\
0.25 * * * \\
0.25\end{array}$ & $\begin{array}{l}\text { rho: } \\
-0.03\end{array}$ \\
\hline $\begin{array}{l}\text { acetic acid } \\
\text { conc. }\end{array}$ & (6: & re & r. & \% & (4) & 4 & X & . & 4 & 箱 & (X) & (1) & (2) & 3) & ( & & $\begin{array}{l}\text { rho: } \\
0.19 * * *\end{array}$ & $\begin{array}{l}\text { rho: } \\
0.26 * \star\end{array}$ \\
\hline $\begin{array}{l}\text { glycerol } \\
\text { conc. }\end{array}$ & (1) & (6) & X & X & ( & 6) & \% & 3 & X & 14 & 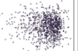 & 35 & (6) & - & 1) & W & & $\begin{array}{c}\text { rho: } \\
0.222 * t\end{array}$ \\
\hline $\begin{array}{l}\text { SO2 } \\
\text { conc. }\end{array}$ & 1) & , & \% & ty & 4 & ) & X. & (6) & 1) & ) & 3 & (5) & (2) & r & ) & - & 3) & \\
\hline & 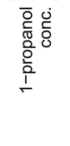 & 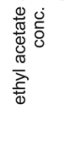 & 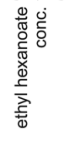 & 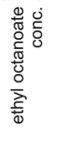 & 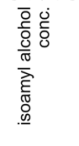 & 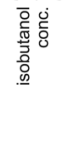 & 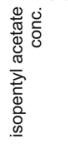 & 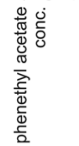 & 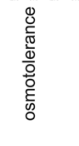 & 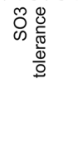 & 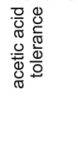 & 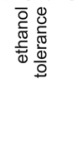 & 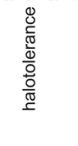 & 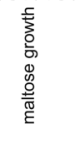 & 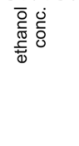 & 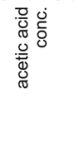 & 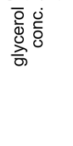 & ర̃ \\
\hline \multicolumn{19}{|c|}{$\begin{array}{l}\text { Fig. } 1 \text { Phenotypic variance across } 1125 \mathrm{~F}_{6} \text { hybrids derived from a cross between S. cerevisiae strains RM11-1a and YJM975a [34]. Each of the } 987 \\
\text { hybrids that did not display a growth defect on maltose was tested in conditions mimicking industrial beer fermentation ( } 16^{\circ} \mathrm{P} \text { at } 20^{\circ} \mathrm{C} \text { for } 7 \text { days), } \\
\text { after which concentration of primary (ethanol) and secondary metabolites (glycerol, acetic acid, higher alcohols and esters) were determined } \\
\text { for each sample. In addition, the growth of all } 1125 \mathrm{~F}_{6} \text { segregants was measured in the presence of high osmolarity (sorbitol), salt, acetic acid, } \\
\text { ethanol, acetic acid and sulfite }\left(\mathrm{SO}^{2-}\right) \text {. Spearman rank correlations were calculated for each pair of phenotypes, rho values are indicated here with } \\
\text { corresponding significance }\left(p<0.10,{ }^{*} p<0.05,{ }^{* *} p<0.01,{ }^{* * *} p<0.001\right)\end{array}$} \\
\hline
\end{tabular}

performance, we asked whether we could identify a relationship between the presence of certain QTNs across the $S$. cerevisiae lineages, and if the ecology of the different strains are subject to enrichment that would offer evolutionary advantages, especially when a founder strain RM11-1a was isolated in a vineyard [4]. To elucidate the ecological relevance of our QTNs, we analyzed their distribution in a set of wholegenome sequences of 1011 $S$. cerevisiae strains, encompassing the full breadth of genetic and phenotypic diversity known for this species to date [37]. We removed 18 QTNs for which we were unable to consistently map the variant position across the 1011 strain collection. 54 QTNs are rare (present in $<1 \%$ of the 1011 strains): either unique to the parental strains, or shared by at most a small number of related strains (Fig. 2C). The contribution of low-frequency alleles confirms previous findings [38], and suggests that a substantial fraction of the missing heritability of complex traits 
might be explained by polymorphisms segregating at low frequency within populations and rare mutations might play a crucial role in modulating the phenotypic landscape within S. cerevisiae as previously reported [37].

By contrast, 78 QTNs are widely spread across the population and shared by $90 \%-99 \%$ of the strains in the collection (Fig. 2C).This is in line with the hypothesis of a single out-of-China origin for this species followed by geographical differentiation, human-associated admixture and several independent domestication events [37]. Next, we analyzed the distribution of all the identified QTLs over the S. cerevisiae natural diversity (as represented by the collection of 1011 sequenced strains). We included all candidate QTLs regardless of whether they were resolved to a single-nucleotide level. A proxy for allelic effects is shown in Fig. 2D as the difference in the mean of a given phenotype between the population of segregants containing either of the parental allele (i.e., distance between the two alleles in Fig. 2D). For clarity, only the 5 QTLs that were predicted to have the strongest effect size are shown in Fig. 2E for each trait. Both of the causal QTNs that were experimentally confirmed (i.e., V5462 in ethanol concentration and V11573 for acetic acid concentration; see below) appear to be rare mutations, accounting for less than $1 \%$ of the total genotyped alleles (Fig. 2E).

As might be expected, several QTLs are present across closely related lineages, indicating a shared evolutionary history and/or admixture between strains from different genetic backgrounds. For instance, the YJM975 $\alpha$ allele of the V7151 QTL for halotolerance has been detected in most of the non-Asian lineages with the exception of the "Alpechin" and "French Guiana human" lineages. The RM11-1a allele of the V6871 QTL associated with 1 -propanol production is mostly observed in Chinese, Taiwanese and Malaysian strains. Other QTLs are unique to a specific lineage and often segregate at low frequency within the population, suggesting a single emergence event in one or few related strains following lineage diversification. For instance, the RM11-1a allele linked to production of phenethyl acetate was detected only in the
African Palm Wine lineage and the RM11-1a allele of the top ethanol tolerance QTL (V5918) was observed only in the Wine European clade and subclade 1. Such events can be caused by either drift or selection but disentangling this process in natural populations remains challenging [39].

Several QTLs are observed in multiple, non-related lineages indicating the presence of multiple emergence events or admixture/crossing. A prominent example is the RM11-1a allele of V10049 QTL for acetic acid tolerance, present in the "Far East Asia" and "Taiwanese" lineages together with the Wine European clade and subclade 2 . The presence of independent emergence events across lineages, might hint at parallel evolution and positive selection, even if neutral drift can never be formally excluded. Interestingly, even though both founder strains are included in the 1011 genome collection (RM11-1a labeled in yellow and YJM975 $\alpha$ blue in the respective clade, Fig. 2E), there are multiple alleles without a single appearance across the whole collection (e.g., V2971 from ethyl acetate and V8458 of halotolerance), suggesting novel variants arose in the founder strains used in this study. Moreover, we observed that $22 \%$ of the QTLs $(N=147)$ are multiallelic across the 1011 genome collection, i.e., show alternative alleles other than the two genotypes found in the parental strains that were used in the cross.

It is tempting to use methods like the McDonaldKreitman test to further investigate whether any of the QTNs show signs of positive or negative selection. However, these methods need to be approached with care, since they assume difference in functional importance between synonymous and non-synonymous mutations $[40,41]$. It is becoming increasingly clear that causal variants can encompass a wide array of molecular variation including synonymous and intergenic mutations $[34,36]$. The level of polymorphism within species and the divergence is also heavily influenced by demography and the local adaption which varies from lineage to lineage and very little is currently known [41]. In a normal case, the stronger representation of a rare allele in certain lineages

\section{(See figure on next page.)}

Fig. 2 Overview of the identified QTLs underlying 18 industrially relevant phenotypes. A Number of causal variants of each functional class identified for each phenotype. B Comparison of the fraction of QTN of each functional class between studies employing the same cross; "Intergenic" includes all single nucleotide polymorphisms at non-coding regions, e.g., promoters, terminators, 5' and 3'UTRs, etc. C Histograms of the number of strains within the 1011 Genome Project yeast collection carrying at least 1 copy of the RM11-1a allele (top) and YJM975a allele (bottom) at the identified QTN across all phenotypes. D Phenotypic effect (as normalized z-scores) of the parental allele for each candidate locus $(N=678)$ and their prevalence in the 1011 sequenced S. cerevisiae strains [37]. The origin of the variants is indicated in blue (RM11-1a) and yellow (YJM975a), and the size of the point represents the frequency of the variant genotype in the 1011 sequenced S. cerevisiae isolates. E Phylogenetic distribution of the top 5 QTLs with strongest average effect on each trait (boxes) by lineage across the 1011 Genome Project yeast collection (phylogenetic tree adapted from [37], mosaic strains were excluded). For each site a distinction is made between QTN (triangle 1nt resolution) and QTL (square from 1 to 1000nt). Size of the circles indicates the fraction of strains within each lineage carrying at least 1 copy of the RM11-1a (blue) and YJM975a (yellow) allele at the specific locus. Red stroke indicates the parental allele with stronger effect on the trait. Lineage assignment is based on [37] and the number of strains is indicated next to the lineage name. RM11-1a and YJM975a lineages are colored blue and yellow, respectively 
A

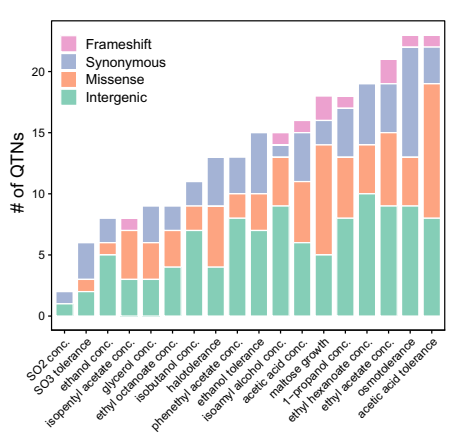

D

$\begin{array}{ll}\text { Parent allele } & \text { Frequency } \\ \text { RM11-1a O YJM975a } & \text { - Less prevalent }\end{array}$

(-propanol conc.

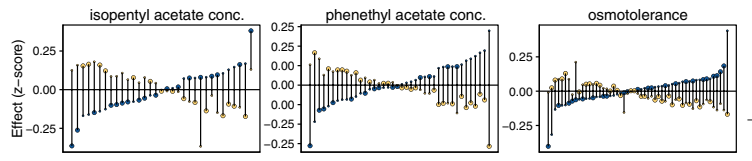

halotolerance

$-$

ethanol conc.
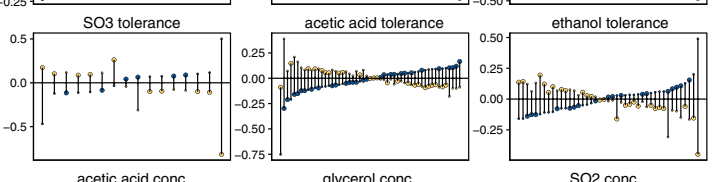

we

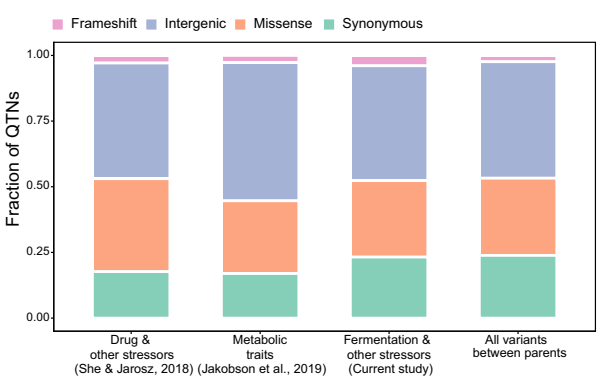

C

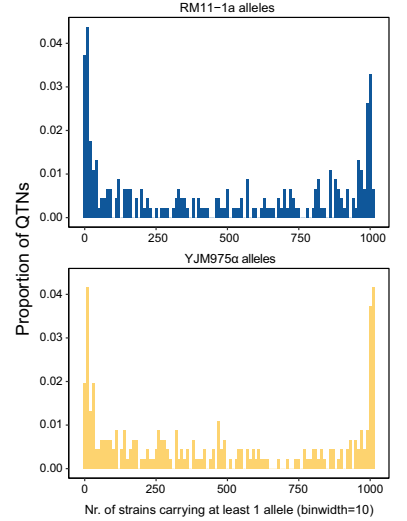

E
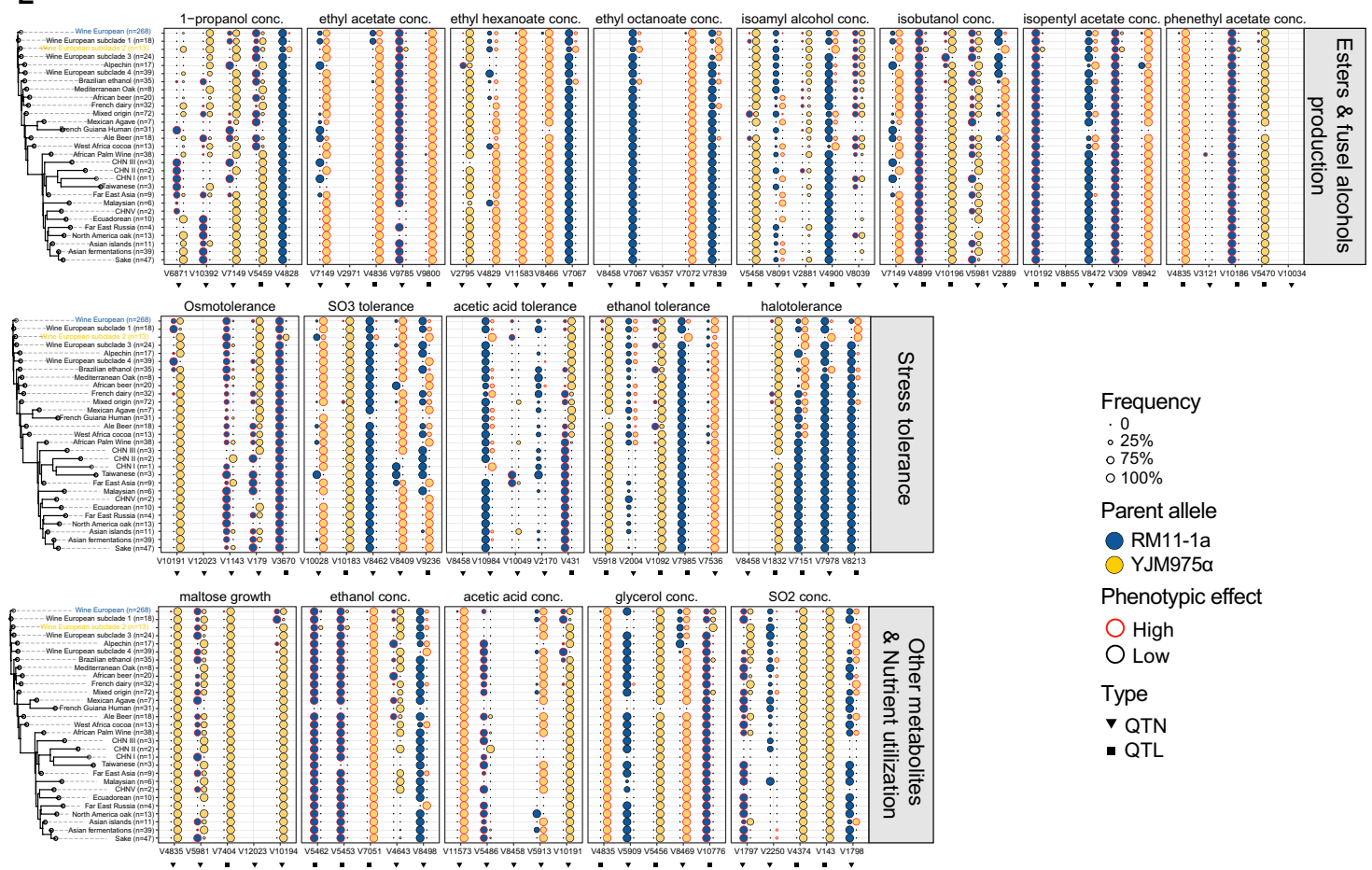

Frequency

.0
$.25 \%$
$075 \%$
$0.100 \%$

$\circ 75 \%$

Parent allele

RM11-1a

YJM975a

Phenotypic effect

$\bigcirc$ High

$O$ Low

Type

$\checkmark$ QTN

- QTL

Fig. 2 (See legend on previous page.) 
may hint to a selection or random genetic drift at play. However, certain phylogenetic clades, like the European Wine lineage, show more rare alleles. After experiencing a strong domestication bottleneck, the wine yeasts clade went through population expansion and accumulated many rare variants $[37,42]$. In addition, although the mosaic strains have been removed from our phylogenetic analyses, many strains might still be the result of ancient admixture which can mislead assumption of parallel evolution if not accounted for.

\section{Experimental confirmation of five selected QTLs}

Compared to the common procedures for QTL mapping in yeast, such as pooled segregant analysis, our pipeline identifies QTLs at the nucleotide-level $(N=254)$, single gene-level (size about $1 \mathrm{~kb} ; N=58$ ) as well as larger genetic loci up to $5 \mathrm{~kb}(N=106)$, which sometimes comprise multiple genes. Still, even these larger loci are considerably smaller than those typically identified in regular QTL approaches where QTL regions are often longer than $10 \mathrm{~kb}$, making it relatively easier to predict which specific variant positions in the region are driving the phenotypic effect.

To confirm the accuracy of our QTL mapping, we selected five QTLs that are predicted to affect multiple industrially relevant phenotypes for further investigation (Table 1 and Fig. 3). Four QTLs were selected because they are pleiotropic, linking to a broad range of different traits, from the production of primary metabolites such as ethanol, glycerol and acetic acid to secondary metabolites including valuable compounds like 1-propanol, ethyl acetate as well as tolerance towards salt and acetic acid (Fig. 3). An additional QTL was selected for its association with the production of isobutanol, which is not only an aroma-active compound that contributes to the flavor of fermented products, but is also being explored as a promising second-generation biofuel [43]. For each of these QTLs, we followed a similar strategy to identify the causative alleles or mutations and their respective phenotypic effects as described in deeper depth in "Materials and methods" section.

\section{Variation in SUC2 causes differences in the production of alcohol and various other metabolites}

One hugely important industrial trait is the ability to convert all available fermentable sugars into ethanol. For this phenotype, we identified 21 QTLs. Among the candidate QTLs, SUC2 stands out because of two reasons. First, this QTL was also mapped to other traits with high significance, including the production of 1-propanol, ethyl acetate, acetic acid, and glycerol (Additional file 2: Table S2). Second, it encodes sucrose invertase, the enzyme that catalyzes the first step in sucrose metabolism, namely the hydrolysis of sucrose into glucose and

Table 1 QTLs called by forward selection regression. List of QTLs with an influence on fermentation parameters, the production of extracellular metabolites and volatile secondary metabolites (Additional files 3, 4, 5)

\begin{tabular}{|c|c|c|c|c|c|c|}
\hline QTL & Trait & Chromosome & Start [bp] & End [bp] & PVAL range & Candidate genes within \\
\hline \multirow[t]{6}{*}{ SUC2 } & 1-Propanol conc & IX & 27,399 & 36,687 & $6.7-37.8$ & NIT1 \\
\hline & Ethyl acetate conc & & & & & SUC2 \\
\hline & Isoamyl alcohol conc & & & & & YIL166C \\
\hline & Ethanol conc & & & & & YIL169C-Gene \\
\hline & Acetic acid conc & & & & & \\
\hline & Glycerol conc & & & & & \\
\hline \multirow[t]{6}{*}{$\mid M A 1$} & Ethyl acetate conc & VII & $1,066,563$ & $1,066,735$ & $7.3-42.0$ & $\mathrm{BlO} 2$ \\
\hline & Isobutanol conc & & & & & IMA1 \\
\hline & Phenethyl acetate conc & & & & & \\
\hline & Acetic acid conc & & & & & \\
\hline & Glycerol conc & & & & & \\
\hline & Maltose growth & & & & & \\
\hline \multirow[t]{3}{*}{ ALD6 } & Ethyl octanoate conc & $\mathrm{XVl}$ & 432,771 & 434,477 & $8.1-112.4$ & ALD6 \\
\hline & Isoamyl alcohol conc & & & & & \\
\hline & Acetic acid conc & & & & & \\
\hline \multirow[t]{5}{*}{ URA5 } & Ethyl octanoate conc & $X I I I$ & 56,990 & 56,990 & $10.5-115.2$ & URA5 \\
\hline & Acetic acid conc & & & & & \\
\hline & Acetic acid tolerance & & & & & \\
\hline & Ethanol tolerance & & & & & \\
\hline & Halotolerance & & & & & \\
\hline URK1 & Isobutanol & XIV & 646,585 & 648,789 & 8.2 & URK1 \\
\hline
\end{tabular}




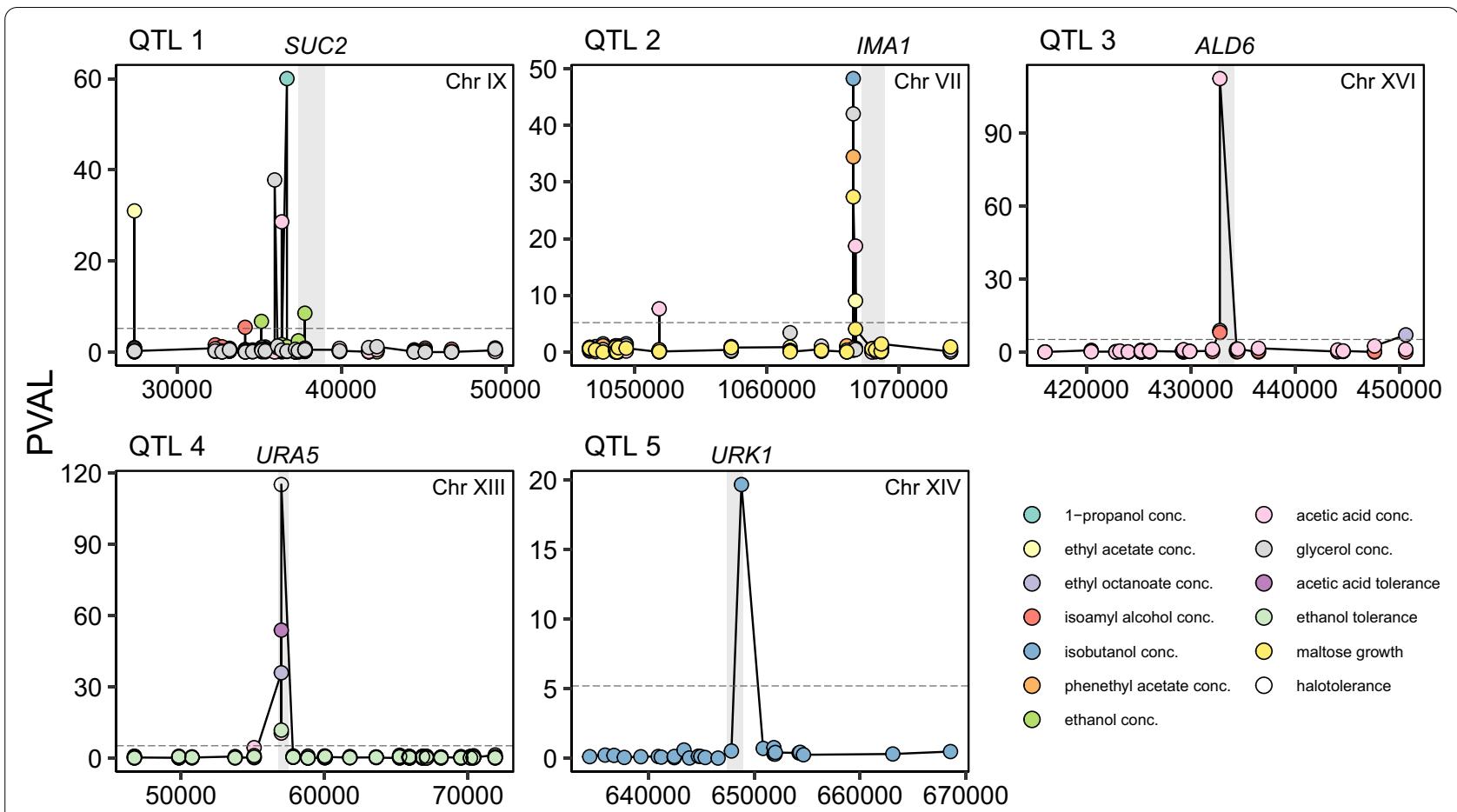

Fig. 3 Genome-wide mapping identifies five candidate loci at single-nucleotide resolution that are predicted to affect multiple industrially relevant phenotypes. PVAL ( $-\log _{10}$ transformed $p$-value from the forward selection) and the chromosomal position are shown for the variants centered around each candidate QTL (indicated in the grey rectangle). The threshold for selecting candidate variants is shown as dotted line (PVAL $=5.2$ )

fructose. Hence, it is tempting to speculate that variation in SUC2 could lead to different efficiencies in sucrose metabolism, which would in turn explain the observed differences in the production of primary (ethanol) and certain secondary metabolites. In line with this hypothesis, deletion of SUC2 in strain RM11-1a led to $6.5 \%$ reduction $(p<0.0001)$ in the final ethanol concentration in the sample, while no effect was observed in strain YJM975 $\alpha$ (Fig. 4). Similarly, SUC2 deletion also led to reduced formation of 1 -propanol $(-10.8 \% ; p \leq 0.001)$, ethyl acetate $(-17.9 \% ; p \leq 0.001)$, acetic acid $(-16.5 \%$; $p \leq 0.01)$, and glycerol $(-10.8 \% ; p \leq 0.0001)$ in RM11-1a, but had no effect in the strain YJM975 $\alpha$, except for the formation of 1-propanol (+8.6\%; $p \leq 0.01)$ (Fig. 4). As the deletion of SUC2 in YJM975 $\alpha$ did not result in significant changes in most of these metabolites, the SUC2 allele of YJM975 $\alpha$ is likely to be a loss-of-function allele, rendering the cells with inferior efficiency in sucrose metabolism as compared to that of RM11-1a.

Next, we attempted to identify the exact causative genetic variation in $S U C 2$ that is responsible for the observed phenotypes. Our QTL pipeline highlighted one frameshift variant in the ORF of SUC2 gene $\left(S U C 2^{394 \Delta}\right)$ with high significance (PVAL $\left.>5.2\right)$ from several traits. However, since the mapping analysis cannot unambiguously distinguish this variant from a nearby variant located at position -6 in the promoter of $S U C 2$, we also included this variant for our validation (Fig. 5A). Although segregants inheriting the entire haplotype block (both $S U C 2^{-6 \mathrm{~A}}$ and $S U C 2^{394 \mathrm{~A}}$ ) from RM11-1a produced higher amounts of ethanol (Fig. 5B), we confirmed that this frameshift mutation is causative for the observed reduction in ethanol production (Fig. 5C). This variant rests immediately at 5' of Suc2's catalytic site [44], and might influence the affinity of the enzyme, as residual sucrose was detected in the finished fermentation sample (Additional file 1: Figure S3). Moreover, it has an opposing effect on the formation of acetic acid and glycerol (Additional file 1: Figure S4), showing its pleiotropy. The fact that the truncated SUC2 allele affects multiple phenotypes is perhaps not surprising, as mutations in this gene affect the total amount of carbon that can be metabolized, thus influencing primary and secondary metabolite production, as well as other traits that may be indirectly linked to growth and metabolism.

To test if the truncated SUC2 allele leads to reduced ethanol production in other strains containing an intact SUC2 allele, we introduced the SUC2 ${ }^{394 \Delta}$ frameshift variant in three diverse industrially relevant strains: (i) Ethanol Red (an industrial bioethanol strain; (ii) CEN. PK (a common chassis strain for heterologous production of high-value compounds) and (iii) S. cerevisiae var. 


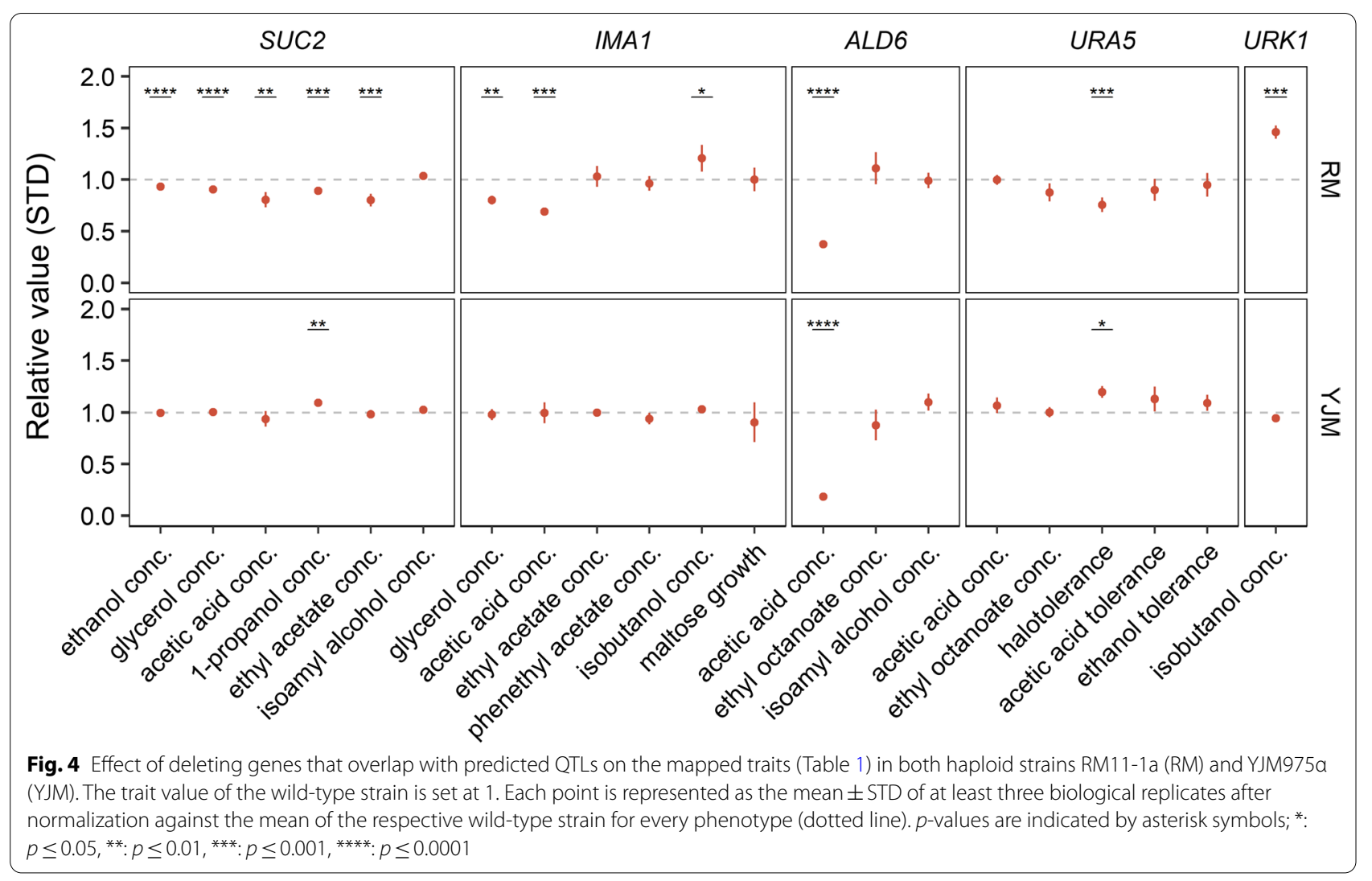

boulardii (a strain often referred to as $S$. boulardii, and commercially used as probiotic). Introduction of the frameshift variant indeed led to reduced ethanol formation in all three strains (Fig. 5D), substantiating the effect of a truncated $S U C 2$ allele on ethanol formation. Next, we investigated whether other candidate QTLs that were mapped to ethanol production would interact with $S U C 2$, however, we did not observe a clear network among these mapped QTLs for ethanol production (Fig. 5E).

YJM975a contains incomplete IMA1 locus, causing reduced maltose utilization and altered formation of certain metabolites

Within QTL2, linked to several traits such as glycerol and acetic acid production as well as maltose utilization, several polymorphisms were identified in the intergenic region between $B I O 2$ and IMA1 genes (approximately $2.2 \mathrm{~kb})$ that are located within the subtelomeric region of chromosome VII (Fig. 3C). While deletion of BIO2 in either of the strain RM11-1a and YJM975 $\alpha$ did not affect any of the phenotypes to which the QTL was linked (data not shown), the deletion of ima1 in RM11-1a led to reduced production of glycerol $(-21.7 \% ; p \leq 0.01)$ and acetic acid $(-30.8 \% ; p \leq 0.001)$, and increased production of isobutanol. By contrast, IMA1 deletion in strain YJM975 $\alpha$ did not result in any significant changes (Fig. 4).

The IMA1 gene encodes for the major isomaltase required for isomaltose utilization, which also exhibits $\alpha-1,2$ glucosidase activity on sucrose and kojibiose $[45,46]$. Near the IMA1 locus, one intergenic variant $\left(I M A 1^{+659 G>C}\right)$ was predicted to influence several traits with strong significance (PVAL $>50$ ). Comparison of the IMA1 locus between strain RM11-1a and YJM975 $\alpha$ revealed one missense mutation $\left(I M A 1^{1007 \mathrm{~A}>\mathrm{T}}\right)$, which has been reported to have a deleterious effect on the growth on raffinose, sucrose and maltose [36]. Yet, swapping either of the two variants between the two strains did not lead to any significant change in the phenotypes that were observed in the imal deletion mutant (Additional file 1: Figure S5). However, a closer evaluation of the YJM975 $\alpha$ genome sequence revealed that it lacks approximately $8 \mathrm{~kb}$ of the genomic region directly upstream of $I M A 1$, including part of the 5'end of $I M A 1$ (207 bp) as well as the entire coding regions of MAL13, encoding the activator protein that activates the permease and hydrolase when substrate is present, and MAL11, encoding the maltose/isomaltose permease [47]. This 8 - $\mathrm{kb}$ deletion segregates in the $\mathrm{F}_{6}$ progeny (Additional file 1: Figure S6A) and correlates with the growth 


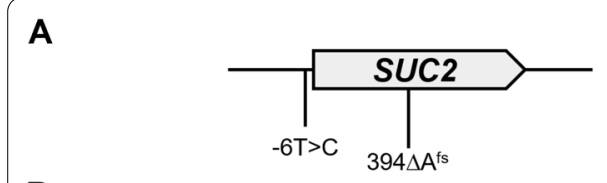

B

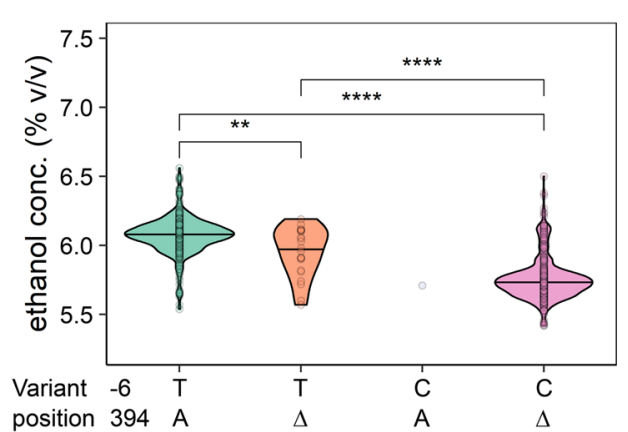

D

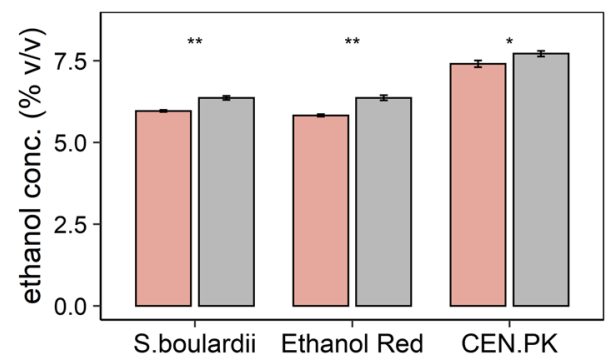

C
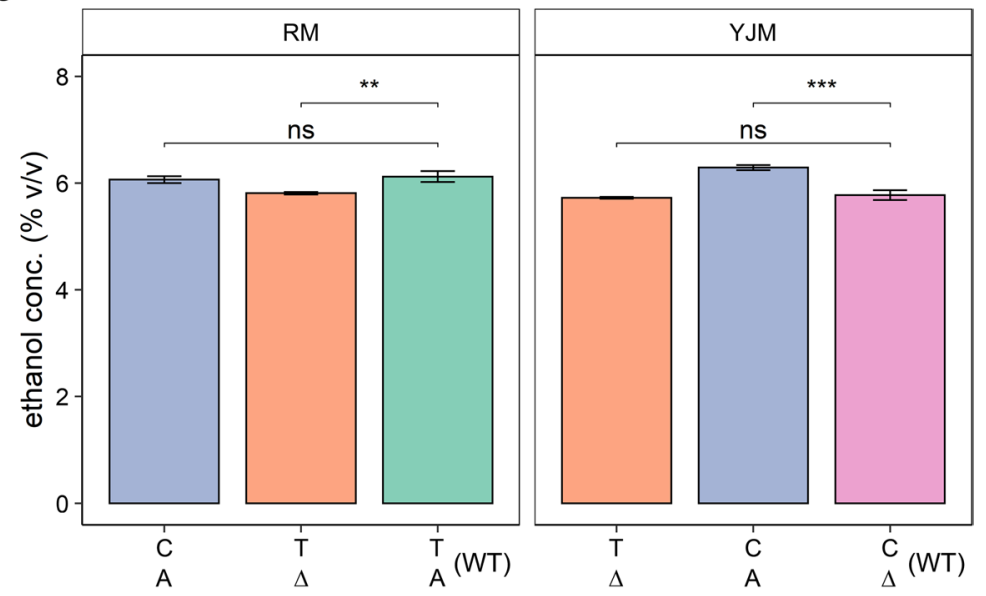

E

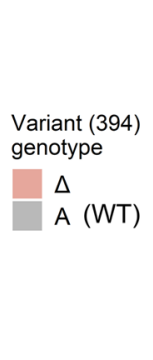

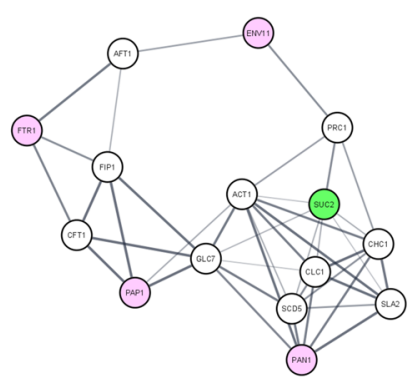
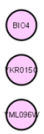

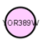

Fig. 5 Identification of the causal variant of reduced ethanol production in the SUC2 locus. A Candidate variants in SUC2. B Meiotic crossovers within the SUC2 locus in the $\mathrm{F}_{6}$ segregants. Swapping the intergenic variant (-6) yields minor phenotypic effect, whereas swapping the true causal variant (394) yields the same major effect as swapping the entire haplotype block. C Ethanol concentration at the end of fermentation (16 $\mathrm{P}$ ) of the wild type (WT) strain RM11-1a and YJM975a and the respective variant-swapped mutants. D Ethanol concentration at the end of fermentation $\left(16^{\circ}\right.$ P) of the wild-type strain S. boulardii, Ethanol Red and CEN.PK and the SUC2 ${ }^{3944}$ variant mutant. E Interaction network of SUC2 (green node) and genes whose coding sequence are altered by variants that were identified for ethanol concentration phenotype (pink node). The thickness of the edges represents the confidence score associated with the interaction as determined by STRING. Data are shown with mean $\pm S T D ; P$-values are indicated with the level of significance (ns: not significant, ${ }^{*}: p \leq 0.05,{ }^{* *}: p \leq 0.01,{ }^{* * *}: p \leq 0.001,{ }^{* * * *}: p \leq 0.0001$ )

on maltose (Additional file 1: Figure S6B), with segregants containing the intact MAL-IMA1 locus showing more efficient growth on maltose, indicating that this structural variation is at the heart of this QTL.

\section{Variation in ALD6 drives changes in acetic acid production}

For QTL3, deletion of ALD6 resulted in significantly reduced acetic acid production in both parental strains, by $63.2 \%$ in RM11-1a $(p \leq 0.0001)$ and by $81.7 \%$ $(p \leq 0.0001)$ in YJM975 $\alpha$ (Fig. 4). ALD6 encodes cytosolic aldehyde dehydrogenase, an enzyme required for the conversion of acetaldehyde to acetate. Our QTL pipeline linked the changes in acetic acid production to a missense variant $\left(A L D 6^{184 A>C}\right)$ located in the $A L D 6$ ORF (Fig. 6A). Segregants inheriting the haplotype from RM11-1a (ALD6 ${ }^{184 C}$ ) produced higher amounts of acetic acid compared to the ones containing the YJM975 $\alpha$ allele $\left(A L D 6^{184 A}\right)$ (Fig. 6B). Swapping the specific alleles between RM11-1a and YJM975 $\alpha$ led to a $22.3 \%(p \leq 0.05)$ reduction in acetic acid formation in RM11-1a, and a 22.0\% $(p \leq 0.001)$ increase in YJM975 $\alpha$, confirming that this variation is indeed the driver in this QTL (Fig. 6C).

To assess the background-specificity of the mutation, we introduced the $A L D 6^{184 C}$ variant in strain Ethanol $\operatorname{Red}^{\circledR}$ and strain CEN.PK as well as in strain S. boulardii, which all carry the $A L D 6^{184 A}$ allele. Especially for this last strain, increased acetic acid production would be hugely beneficial, since its probiotic effect is at least partly attributed to the production of acetic acid, which can affect the growth of other microbes in the gastrointestinal tract [48]. Introduction of the mutation indeed led to increased acetic acid formation in all three strains, most notably in Ethanol Red (79\%) and S. cerevisiae var. boulardii (39\%), showing that some of the QTLs identified in this study could serve as a basis to further improve industrial strains (Fig. 6D). 


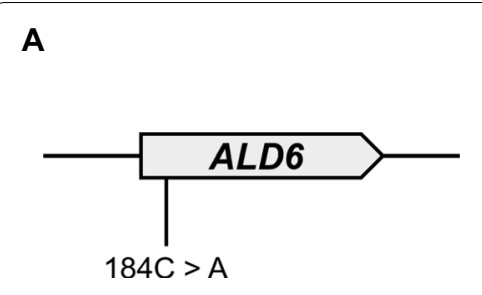

B acetic acid ( $\mathrm{g} / \mathrm{l})$

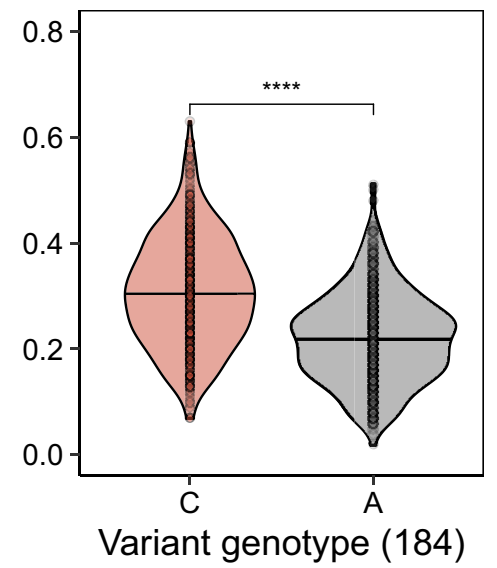

C

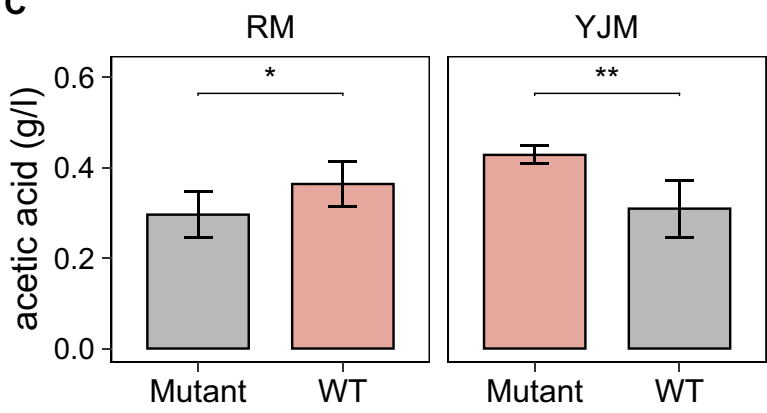

D

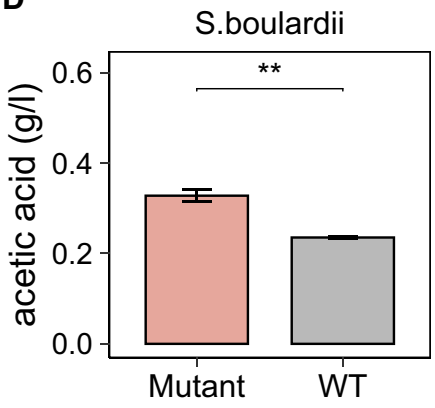

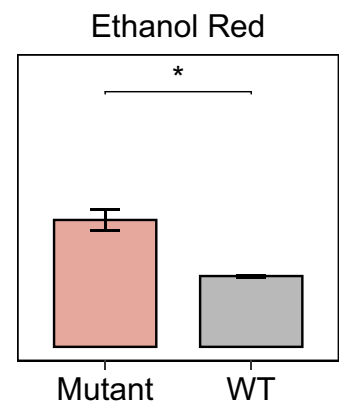

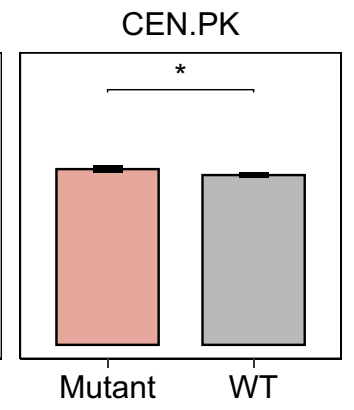

Fig. 6 Identification of the causal variant underlying differences in acetic acid production A Candidate variants in ALD6. B Meiotic crossovers within the ALD6 locus in the $\mathrm{F}_{6}$ segregants. C Acetic acid production of the variants swapped in the parent strain RM11-1a and YJM975a, and $\mathbf{D}$ in S. boulardii, Ethanol Red, and CEN.PK. Error bars represent standard deviation of 3 biological replicates. $P$-values are indicated with the level of significance $\left(^{*}: p \leq 0.05,{ }^{* *}: p \leq 0.01,{ }^{* * *}: p \leq 0.001,{ }^{* * * *}: p \leq 0.0001\right)$

\section{Some QTLs can be linked to a gene, but not an exact allele}

Deletion of URA5 led to salt sensitivity in strain RM111a, while improving salt tolerance in strain YJM975 $\alpha$ (Fig. 4), suggesting that URA5 is the causal element in QTL4 for the differential halotolerance between the two strains. Further analysis identified a missense variant within URA5 (URA $\left.5^{266 G>T}\right)$, which encodes for the major orotate phosphoribosyl transferase (OPRTase), catalyzing the fifth enzymatic step in de novo biosynthesis of pyrimidines [49]. However, despite several attempts we could not swap the missense variant between the parent strains and were therefore unable to confirm whether this point mutation drives the QTL. In addition to halotolerance, the URA5-related QTL is also linked to other traits such as ethanol tolerance (Fig. 3). It is likely that this pleotropic effect is exerted through the biosynthetic pathway of pyrimidines. Indeed, URA5 catalyzes the step upstream of $U R A 3$ which has already been connected to ethanol tolerance as the causal QTL in a cross between a bioethanol production strain and a laboratory strain [30].

Similarly, for QTL5, deletion of URK1 resulted in significantly increased production of isobutanol by $47.2 \%$ in RM11-1a (Fig. 4). URK1 encodes pyrimidine kinase, an enzyme involved in the deoxyribonucleotide salvage pathway. Interestingly, the deletion of $U R K 1$ in Ethanol Red and CEN.PK also led to increased production of isobutanol, while no effect was observed in S. boulardii (Additional file 1: Figure S7B), suggesting that there might be complex background interactions that further influence this trait. Mapping analysis identified two missense variants in the ORF of URK1 (URK1 $1^{412 A>C}$ and $\left.U R K 1^{1358 G>A}\right)$. However, swapping either of the two variants between strain RM11-1a and YJM975 $\alpha$ did not lead to any significant change in the production of isobutanol (Additional file 1: Figure S7A).

\section{Discussion}

Our study identified 678 putative QTLs that are predicted to drive differences in various industrially relevant phenotypes, including resistance to various stress factors and the production of primary and secondary metabolites. Further analysis of 5 QTLs showed that in each case, we were able to link a certain gene located within the QTL to some or all phenotypes that are influenced by the QTL. In two cases, the analysis pipeline correctly predicted specific polymorphism at single-nucleotide 
level as the driver of the QTL. In another case, the QTL proved to be linked to a large segmental deletion. In the two remaining cases, we were able to link a specific gene to the respective traits, but unable to pinpoint the exact causative mutation.

\section{LEU2 auxotrophy influences the formation of fusel alcohols and esters}

The use of auxotrophic marker in strain construction rests on the assumption that in medium where the auxotrophy is complemented by supplementation of a specific compound, the phenotypic effect is minimal. However, this assumption may not hold true when the auxotrophically required compounds interfere with the biosynthesis of other metabolic compounds and/ or influence the expression of specific genes [50]. We also observed this in our data, where the specific growth rate of wild-type $S$. cerevisiae is reduced when only leucine is supplemented in the synthetic medium without the addition of isoleucine or valine, which is in line with the previously reported influence of leucine on the biosynthesis of the other two branched-chain amino acids [51]. Apart from the impact on physiology, amino acid autotrophy has also already been linked to a number of industrially relevant traits. For example, URA3 was identified as the causal QTL for ethanol tolerance in a cross between a bioethanol production strain and a laboratory strain [30]. The same auxotrophy also leads to the difference in maximal ethanol accumulation capacity between a sake and a laboratory strain [52]. Similarly, leucine autotrophy has been suggested to affect the cell's resistance to rapamycin in the same set of segregants used in this study [34]. Here, we show that leucine auxotrophy imposes a strong effect on fermentation phenotypes, and specifically aroma formation, because metabolism of leucine in yeast is tightly linked to the formation of various flavor-active compounds [53], including organic acids, aldehydes, and higher (fusel) alcohols [54]. Several of these higher alcohols are currently explored as biofuels [55], but they are also the main precursors of acetate esters, which are important contributors to the flavor and aroma of alcoholic beverages [56, 57]. In particular, LEU2 encodes for beta-isopropylmalate dehydrogenase, involving in the conversion of $\alpha$-ketoisovalerate (KIV) to $\alpha$-ketoisocaproate (KIC) which is a direct intermediate in the biosynthetic pathway of isoamyl alcohol. As a result, the production of isoamyl alcohol and its derivative ester isopentyl acetate are prominently affected in the $F_{6}$ progeny by the segregation of leucine auxotrophy (Additional file 1: Figure S1). The fundamental impact of leucine auxotrophy on the traits measured in the current study highlights how changes in the expression of a single auxotrophic marker can resonate throughout the entire metabolic network and lead to an altered pool of metabolites. Therefore, great care should be exercised in the design of the experimental setup to mitigate possible drawbacks from the application of these auxotrophic markers (see also [50]).

\section{Abundant pleiotropic interaction among identified QTLs}

Pleiotropy refers to the effect of a particular allele on more than one phenotype. Our data indicate that pleiotropy is common across traits examined in this study. At the molecular level, roughly $39 \%(N=267)$ of the QTLs are linked to more than one trait (Additional file 2: Table S3). This may also at least partly explain why certain phenotypes are correlated (Fig. 1). We define a pleiotropic QTL as a variant that is linked to more than one phenotype, or when several variable sites within one QTL region (with a maximal dimension of $10 \mathrm{~kb}$ ) are linked to different traits. This is particularly important for the identification of the segmental deletion at the IMA$M A L$ locus as well as the precise validation of the causal frameshift variant located in $S U C 2$. In the former case, as the deleted genomic region prevents association of precise variants within this locus, several intergenic variants close to the deleted locus were linked to multiple traits without nucleotide-level resolution. In the latter case, the close vicinity of the frameshift variant $S U C 2^{394 \Delta A}$ and an intergenic variant that locates $400 \mathrm{bp}$ away prevents frequent recombination between the haplotype block in the $\mathrm{F}_{6}$ segregants, leading to multiple candidate variants that could not be unambiguously distinguished.

Swapping of the $S U C 2^{394 \Delta A}$ allele between the founder strains results in a concerted change in the production of ethanol, glycerol, and acetic acid. This interconnection is in line with previous reports [58-60]. One possible explanation that has been suggested is that while alcoholic fermentation is redox neutral, the biomass formation generates surplus $\mathrm{NADH}$, requiring other pathways for the regeneration of $\mathrm{NAD}^{+}$to maintain flux through glycolysis. In response, cells synthesize glycerol to regenerate $\mathrm{NAD}^{+}$, however, excess $\mathrm{NAD}^{+}$may be formed, which is then balanced through the formation of acetic acid from acetaldehyde to convert $\mathrm{NAD}^{+}$back to NADH that functions as redox sink.

\section{Structural variation affects multiple fermentation-relevant traits}

In all-malt brewer's wort, maltose typically accounts for $60 \%$ of the total fermentable sugar for the yeast and efficient maltose utilization is therefore crucial for beer production. Maltose metabolism requires 3 key genes, encoding maltase (MALS), a transporter for maltose (MALT) and a positive regulator (MALR) [61]. These 3 genes typically cluster together in the genome in 
subtelomeric regions, and most genomes harbor several such gene clusters, scattered across different chromosomes [46, 62]. In some cases, the elevated rate of mitotic and meiotic recombination in the subtelomeric region may also lead to chromosomal rearrangements in these $M A L$ loci. For instance, the impaired growth phenotype on maltose is linked to the deleted genomic region (ca. $8 \mathrm{~kb}$ ) at the $I M A 1-M A L 1$ locus in the genome of YJM975 $\alpha$. This structural variation highlights the relevance of the copy number of the MAL (maltose) gene with utilization of maltose, which is in accordance with the findings of [1], where yeast domestication led to more efficient fermentation of specific carbon sources like maltose through mutations and duplications of the $M A L$ genes [1]. In addition to maltose growth phenotype, the missing locus also links to other traits, including the production of ethyl acetate, isobutanol, phenethyl acetate, acetic acid, and glycerol (Additional file 2: Table S2). The paramount impact of maltose utilization on the production of metabolites was already anticipated at the start of the project, and all $\mathrm{F}_{6}$ segregants were therefore propagated in YPMaltose for 3 days prior to the main fermentation to detect major growth defects. The profound pleiotropic effect of this locus calls for a more stringent screening scheme, which would inevitably be at the cost of reducing mapping resolution.

While absence of the entire IMA1-MAL1 locus is linked to impaired maltose utilization, deletion of the IMA1 locus by itself also leads to multiple phenotypes in RM11-1a, including the reduced formation of glycerol, acetic acid and isobutanol (Fig. 4). The pleiotropic effect of IMA1 may be explained by the substrate specificity of the corresponding Imal enzyme, which shows activity towards sugar oligomers with $\alpha-1,6$ - or $\alpha-1,2$-glycosidic linkages, such as isomaltose, sucrose and dextrin $[45,63]$. Specifically, IMA1 has been suggested to have a minor (yet relevant) activity towards sucrose in the absence of SUC2, which encodes the major sucrose invertase [64]. Although these $\alpha$-glucosides are present in relatively small quantity in the wort [65], the assimilation of these sugars impacts beer quality and affects the production of flavor-active metabolites. For instance, significant differences in volatile profiles were obtained as different carbohydrates were fermented by yeast [66].

\section{Prevalence of the identified causal QTNs across a diverse strain collection}

The distribution of the different QTNs that were identified in this study closely resembles the distribution of all SNPs across S. cerevisiae strains (Fig. 2). This might suggest that many QTNs only have relatively minor, nearneutral effects and that are not under strong (negative or positive) selection. Still, our analyses show that a few variants, such as for example the $S U C 2^{394 \Delta A}$ frameshift mutation, do have major phenotypic effects. From an ecological standpoint, the $S U C 2^{394 \Delta A}$ frameshift allele renders cells with reduced ability to utilize a carbon source (i.e., sucrose), so such deleterious alleles are predicted to be rare when natural selection operates on the QTL. Recurring deleterious alleles is an important source of genetic variation which can result from de novo mutation or transfer of the alleles from a nearby population through hybridization or horizontal gene transfer. However, it is not trivial to classify a given mutation as negative because it might be deleterious in existing environments or genetic backgrounds but beneficial in other ecological or genetic contexts. As an example, the $A L D 6^{184 A>C}$ variant resulted in elevated formation of acetic acid production, which can help suppressing growth of competing bacteria in complex microbial communities [48], but might be detrimental for the yeast's fitness in man-made, pure-culture fermentation environments [67].

\section{Context-dependent QTL}

Isobutanol is a promising second-generation biofuel [43], which is produced by yeasts in small amounts through the degradation of valine via the Ehrlich pathway [54], and multiple metabolic engineering approaches have been proposed to boost production $[68,69]$. Our results show that some QTLs also influence the natural production of isobutanol, and this led us to ask whether these could be used to increase the production of this metabolite. We verified URK1 as the causal QTL for the production of isobutanol. Moreover, deletion of URK1 resulted in similar phenotypes across different strains, suggesting the effect of this QTL is not context-specific and could be used to boost isobutanol production in industrial strains. Urk1 is a uridine kinase involved in the pyrimidine salvage pathway, which is responsible for the biosynthesis of pyrimidines required for the synthesis of nucleic acid and amino acids [70]. However, a direct link between Urk1 and isobutanol production has never been reported.

\section{Conclusions}

In the current study, we identified 678 candidate variants for 18 industrially relevant traits using an inbred yeast population consisting of $1,125 \mathrm{~F}_{6}$ progeny of a cross between two strains, RM11-1a and YJM975 $\alpha$. Experimental validation confirmed the contribution of five genetic loci, of which two were pinpointed to the singlenucleotide level. The identified loci consisted of both coding and intergenic regions, and comprised a broad range of different types of mutations, ranging from structural variation to InDels and SNPs. Interestingly, the effect of some industrially relevant QTLs was consistent 
in different genetic background. For example, transferring the $A L D 6^{184 A>C} \mathrm{QTN}$ in a probiotic strain increased its acetic acid formation (and thus possibly also its probiotic effect), while deletion of URK1 led to increased isobutanol production in an established biofuel strain. On the other hand, we also show that many of the loci affect multiple phenotypes, which implies that engineering these QTLs into a strain will likely also result in multiple changes, a factor that has not received sufficient attention in studies promoting gene editing of industrial yeasts. Together, our results demonstrate the effectivity of the described approach to detect causal variants for complex traits and open new avenues for optimizing strains in a broad range of biotechnological applications.

\section{Materials and methods Yeast strain}

The yeast strains used and constructed in the study are listed in Additional file 2: Table S4. The parent strain RM11-1a and YJM975 $\alpha$ as well as their $1,125 \mathrm{~F}_{6}$ haploid progeny were obtained from Dr. Jarosz group [34]. Saccharomyces boulardii was isolated from lyophilized cells that is available in the market as probiotics in capsules (BIOCODEX, BENELUX). The strains were routinely maintained on solid YPD medium containing $10 \mathrm{~g} \mathrm{~L}^{-1}$ yeast extract, $20 \mathrm{~g} \mathrm{~L}^{-1}$ peptone, $20 \mathrm{~g} \mathrm{~L}^{-1}$ glucose, and $15 \mathrm{~g}$ $\mathrm{L}^{-1}$ agar. Frozen stocks of all strains were maintained at $-80^{\circ} \mathrm{C}$ using a glycerol-based storage medium $\left(20 \mathrm{~g} \mathrm{~L}^{-1}\right.$ Bacto peptone, $10 \mathrm{~g} \mathrm{~L}^{-1}$ yeast extract, $20 \mathrm{~g} \mathrm{~L}^{-1}$ glucose, $250 \mathrm{~mL} \mathrm{~L}^{-1}$ glycerol).

\section{General molecular biology and microbiological techniques} Genomic DNA extraction from yeast was performed using phenol-chloroform-isoamyl alcohol (PCI) according to the method described by [71]. Plasmids were isolated from E. coli $\mathrm{DH} 5 \alpha$ cells from overnight cultures in lysogeny broth (LB) containing $10 \mathrm{~g} \mathrm{~L}^{-1}$ peptone, $5 \mathrm{~g} \mathrm{~L}^{-1}$ yeast extract and $10 \mathrm{~g} \mathrm{~L}^{-1} \mathrm{NaCl}(\mathrm{pH} 7.0)$ with $100 \mathrm{mg} \mathrm{L}^{-1}$ carbinicilin by using the Qiagen Miniprep Kit (Qiagen, Germany). Transformation of yeast cells with plasmids as well as PCR-amplified DNA fragments for genomic integration was performed using LiAc/PEG method described by [72].

\section{Lab-scale fermentation in wort}

Yeast pre-cultures were inoculated overnight at $20{ }^{\circ} \mathrm{C}$ in test-tubes containing $3 \mathrm{~mL}$ of $10 \mathrm{~g} \mathrm{~L}^{-1}$ yeast extract, $20 \mathrm{~g} \mathrm{~L}^{-1}$ peptone, $40 \mathrm{~g} \mathrm{~L}^{-1}$ maltose medium (YPMal). After $16 \mathrm{~h}$ of incubation, $1 \mathrm{~mL}$ of the pre-culture was used to inoculate $50 \mathrm{~mL}$ of the YPMal medium in 250 $\mathrm{mL}$ Erlenmeyer flask and propagated in the same conditions as the pre-culture for 3 days. Notably, $10 \%$ of the segregants showed growth defects during pre-culturing and thus were exempted from further fermentation. The propagated cells were then used for inoculation of the fermentation medium, i.e., $16^{\circ}$ Plato $\left(16^{\circ} \mathrm{P}\right)$ wort prepared by in-house brewery at a pitching rate of $10^{6}$ cells $\mathrm{mL}^{-1}$. A blank wort medium was included in each batch of fermentation. Fermentations were performed in 250$\mathrm{mL}$ Schott bottles with a water lock placed on each bottle and stirred at $150 \mathrm{rpm}$ for 7 days at $20{ }^{\circ} \mathrm{C}$. Weight loss was monitored daily to follow the progress of fermentation. After 7 days, the fermentations were terminated on ice to minimize evaporation of volatile compounds and sampled for analytical analysis. Fermentation of all segregants was individually performed only once.

\section{Analytical methods}

Quantification of yeast aroma production was carried out using headspace gas chromatography coupled with flame ionization detection (HS-GC-FID; Shimadzu, Japan). The GC was calibrated with 8 important aroma compounds, including isobutanol, isoamyl alcohol, ethyl acetate, ethyl hexanoate, ethyl octanoate, isoamyl acetate and phenethyl acetate, using 2-heptanol as the internal standard. Specifications of the GC system and the sample preparation are as described by [73]. Ethanol measurements were performed with the Alcolyzer Beer DMA $4500 \mathrm{M}$ (Anton Paar, Austria). Filtered samples ( $0.15 \mathrm{~mm}$ paper filter) were measured for the level of glycerol, acetic acid and sulfite using Thermo Scientific Gallery discrete photometric analyzer (Thermo Fisher Scientific, USA). Sugar concentrations were determined by Dionex Liquid Chromatography (Thermo Fisher Scientific, USA), which was calibrated for maltose, sucrose, glucose and fructose using raffinose as the internal standard.

\section{Yeast phenotyping}

$\mathrm{F}_{6}$ segregants taken from frozen stock were pinned on solid YPD medium using a Singer ROTOR robotic pinning instrument, with which cells were subsequently transferred to various solid media (YPD with stress agents as indicated, carbon source is $20 \mathrm{~g} \mathrm{~L}^{-1}$ glucose if not otherwise indicated) for phenotyping and incubated at $30{ }^{\circ} \mathrm{C}$ for $48 \mathrm{~h}$. Cells were duplicated on a blank YPD plate (containing no additional stress/agent) for each growth assay to normalize for inherent growth differences. Growth was monitored daily in 1536-spot format by scanning the plates; colony size was quantified by using the programming language $\mathrm{R}$ (www.r-project.org) with package R/gitter v1.1.1 [74]. Prior to data analysis, cell growth was normalized by equating colony sizes of the trait to that of the corresponding blank plate. 


\section{Mapping of the variants}

Phenotypical measurements were corrected for batch effects for visualization purposes by performing stepwise regression for each phenotype with genotypes and fermentation batches as predictors ( $p$ cutoff $=0.01)$. Resulting coefficient estimates were multiplied with measurements from the corresponding batch. Quantitative trait nucleotide (QTN) scores for each phenotype were calculated using the pipeline developed by [34] ( $p$ cutoff $=0.01$, false discovery rate cutoff $\log (p)=5.2$ ). Due to the drastic effect of the LEU2 deletion on general fermentation performance, all subsequent analyses of the traits that are known to be highly linked to LEU2 were performed separately on the segregants with and without the gene.

During the propagation step prior to fermentation, approximately $15 \%$ of the progeny showed compromised growth on maltose $\left(\mathrm{OD}_{600}<1.0\right.$ after 3 days of cultivation in YPMaltose). As this growth defect leads to only partial consumption of the available carbon, which in turn causes large deviations in metabolite production that obscure some of the more subtle differences induced by other QTLs. Segregants showing growth defects on maltose were therefore excluded from experiments aimed at characterizing metabolite production during fermentation, and only the remaining 758 segregants that did not exhibit a growth defect on maltose were included in the analysis pipeline. A set of other phenotypes, most notably resistance to stress factors, were tested in rich YPD medium with glucose as carbon source. These phenotypes are therefore not influenced by maltose consumption, and the data for all 845 prototrophic F6 segregants were used in the analysis pipeline.

\section{Experimental confirmation of five selected QTLs and variant replacements}

Validation of the candidate variants was carried out in two steps. In a first step, we tested the contribution of several candidate genes located within the predicted QTLs to a given phenotype by checking the phenotypic effect of deleting the gene in both parental genetic backgrounds. Apart from their central location in the predicted QTL regions, the target genes were selected either because they contain non-synonymous variants between the parent strains RM11-1a and YJM975 $\alpha$ and/or have a molecular phenotype (enzymatic activity or transcriptional activity) that could be linked to the specific phenotype under investigation. For some QTLs, we performed a second set of experiments where the parental alleles were swapped.

The locus harboring a candidate variant was deleted in both parent strains by genomic integration of a disruption cassette containing the nourseothricin (clonNAT) resistance gene (NatMX). The deletion cassette was obtained by PCR from the plasmid pV1382 (addgene, USA) using primers del_QTL_fw and del_QTL_rv (Additional file 2: Table S5). When phenotypic difference was observed between the constructed mutant and wild-type strain, the candidate variant was subsequently swapped between the parents via CRISPR-Cas9-mediated genome editing. To target each candidate variant, a unique guide RNA (gRNA_QTN_fw; gRNA_QTN_rv) containing plasmid was constructed based on pV1382 as the backbone (Additional file 2: Table S5). Repair fragments (100 bp) containing the parental genotype of each target variant was prepared by annealing primers RF_QTN_fw_parent and RF_QTN_rv_parent (Additional file 2: Table S5) with 50-60 bp extensions homologous to regions up- and downstream of the target locus. To swap the target QTN in the parent strains, the respective guide RNA plasmid and the repair fragments containing the genotype of the counterparts were co-transformed reciprocally. Transgenic strains were selected on YPD solid medium supplemented with $200 \mu \mathrm{g} \mathrm{mL} \mathrm{m}^{-1}$ ClonNAT. The correct constructs of the QTL deletion and QTN swap mutants were verified with PCR and/or Sanger sequencing using primers ver_QTL_fw and ver_QTL_rv (Additional file 2: Table S5).

\section{Genetic mapping for growth differences on maltose}

Copy number and mutations on the MAL genes were identified by using the raw sequencing data from [34]. Briefly, raw reads were trimmed and filtered using Trimmomatic v.0.33 [75], clipping bases with quality score below 20 and discarding reads shorter than $30 \mathrm{bp}$. The quality of the resulting trimmed reads was assessed with FastQC v0.11.8 (http://www.bioinformatics.babraham. ac.uk/projects/fastqc/). Trimmed reads were aligned to S288C reference genome with the Burrows-Wheeler Aligner v.0.7.17 [76], and short variants were detected following the Genome Analysis Toolkit best practices, using GATK v4.0.11 [77]. After variants genotyping, the allelic information for each of the 1,152 samples were extracted using a custom python script. For each sample, IMA1 long and short alleles were identified by assessing the IMA1 locus and upstream region coverage (chromosome VII: 1,069,000-1,076,000 bp) with bedtools v2.29.0 [78]. All results were confirmed by carefully analyzing mapped reads tracks on genome browser IGV v2.4.16 [79].

\section{Analysis of QTL distribution across natural S. cerevisiae strains}

The 1011Matrix.gvcf.gz, containing all SNPs and indels called at the population level for 1011 S. cerevisiae strains, was downloaded from the "The 1002 Yeast Genome 
project" website (http://1002genomes.u-strasbg.fr/files/). For each QTL site identified in this study, the occurrence of the RM11-1a and YJM975 $\alpha$ alleles was counted across strains in terms of number of strains (genotypes) carrying at least 1 copy of the allele, and of number of alleles present in each lineage over the total number of alleles in the called genotypes within the lineage (Info field $=\mathrm{AN}$, VCFv4.1). Sites for which the reference or alternative alleles did not match the RM11-1a and YJM975 $\alpha$ alleles from [34], were excluded from all analyses. Lineage assignment was based on [37] and mosaic lineages were excluded prior calculation of the phylogenetic distribution of the identified QTLs. SnpEff (v4.3) [80] was used to annotate and predict the effect of the variants.

\section{Bioinformatic analysis}

Gene networks representing the most depleted and enriched deletion strains were made in STRING version 11.0 [81] and visualized using Cytoscape version 3.7.1 [82].

\section{Supplementary Information}

The online version contains supplementary material available at https://doi. org/10.1186/s13068-021-02059-w.

Additional file 1. Figure S1. The absence or presence of $L E U 2$ influences the progeny's phenotypes. Segregants with or without LEU2 are labeled in yellow or grey respectively. Figure S2. Fraction of overall QTLS and the top 10 percent QTLs with strongest average effect on the trait compared to the distribution of all variants between the parental strains. Figure S3. Residual sucrose at the end of fermentation (16 $\mathrm{P}) . \mathrm{N}=3$ biological replicates. $P$-values are indicated by asterisk symbols $\left(*: p<=0.05,{ }^{* *}\right.$ $\left.p<=0.01,{ }^{* * *}: p<=0.001,{ }^{* * * *}: p<=0.0001\right)$. Figure S4. Effect of swapping predicted QTL alleles on various phenotypes between the haploid strains RM11-1a (RM) and YJM975a (YJM). A) frameshift variant (394 $\triangle \mathrm{A}^{\mathrm{fs}}$ ) in SUC2 and B) 184A >C in ALD6. Each point is represented as normalized mean \pm STD of at least three biological replicates. $P$-values are indicated by asterisk symbols $\left({ }^{*}: p<=0.05,{ }^{* *}: p<=0.01,{ }^{* * *}: p<=0.001,{ }^{* * * *}\right.$. $p<=0.0001$ ). Figure S5. Swapping of variants that are linked to IMAI between the haploid strains RM11-1a (RM) and YJM975a (YJM) did not result in significant change of the traits where IMA1 was mapped to. Each point is represented as normalized mean \pm STD of at least three biological replicates. $P$-values are indicated by asterisk symbols $\left(^{*}: p<=0.05, * *\right.$ : $\left.p<=0.01,{ }^{* * *}: p<=0.001,{ }^{* * * *}: p<=0.0001\right)$. Figure S6. Correlation between the IMA1-MAL genotype on chromosome VII and growth on maltose as the carbon source. A) Average sequencing coverage of the $F_{6}$ segregants at the IMA1-MAL locus on Chromosome VII. B) Relative growth on maltose of segregants according to their parental genotypes at the IMA1-MAL locus. Figure S7. Identification of the causal variant underlying differences in isobutanol production $\mathbf{A}$ ) Isobutanol production of the variants swapped between the parent strains RM11-1a and YJM975a. B) Isobutanol production of URK1 knockout (KO) and WT strains of S. boulardii, Ethanol Red, and CEN.PK. Error bars represent standard deviations from three biological replicates. $P$-values are indicated by asterisk symbols (*: $\left.p<=0.05,{ }^{* *}: p<=0.01,{ }^{* * *}: p<=0.001,{ }^{* * * *}: p<=0.0001\right)$. Figure S8 Zoom-In subgraph of Fig. 1 (1/2). Figure S9. Zoom-In subgraph of Fig. 1 (2/2). Figure S10. Zoom-In subgraph of Fig. 2 (1/3). Figure S11. Zoom-In subgraph of Fig. 2 (2/3). Figure S12. Zoom-In subgraph of Fig. 2 (3/3).

Additional file 2. Table S1. Mapping analysis of the 18 different yeast traits relevant to industrial fermentations and biotechnological processes of all 1,125 previously sequenced inbred segregants $\left(F_{6}\right)$. Table S2. Mapping analysis of the 18 industrially relevant phenotypes of the prototrophic $F_{6}$ segregants. Table S3. Overview of the identified QTLs, including the analysis of their distribution across natural S. cerevisiae strains (The 1002 Yeast Genome project). Table S4. The yeast strains used and constructed in the current study. Table S5. The oligonucleotides used in the current study.

Additional file 3. Phenotypic measurements of the industrially relevant traits from the 1,125 $\mathrm{F}_{6}$ segregants.

Additional file 4. Data used for plotting the main figures.

Additional file 5. Data used for plotting the supplementary figures.

\section{Acknowledgements}

We thank Christopher M. Jakobson for the technical advice.

\section{Authors' contributions}

SP conducted the QTN mapping as well as generated the multivariate plots. BG designed and implemented the phylogenetic analyses and characterized the variant distributions. ADC implemented the custom code and carried out the genetic mapping for growth differences on maltose. JS, DJ and KV conceived and supervised the study and gave input on the different analyses. $\mathrm{PWH}$ performed and led the experimental work and wrote the first draft of the manuscript. P-JP, FR, JV, BH, JC, RN and VS helped with the experimental work, including the setup and analysis of the 1125 fermentations. All authors contributed to writing and editing of the manuscript. All authors read and approved the final manuscript.

\section{Funding}

J.S. is an FWO postdoc fellow (12W3918N). Research in the laboratory of K.J.V. is supported by VIB, AB-InBev-Baillet Latour Fund, FWO, VLAIO, European Research Council (ERC) Consolidator Grant CoG682009 and ERC PoC Grant 959109-SUPERYEAST). D.F.J. was supported by grants from the National Institutes of Health (DP2-GM119140 and RF1-AG057334) and National Science Foundation (NSF-MCB116762) as well as fellowships from the David and Lucile Packard Foundation and the Bert and Kuggie Vallee Foundation.

\section{Availability of data and materials}

Raw whole-genome sequencing reads of $\mathrm{F}_{6}$ segregants and the 2 parental strains are available at https://www.ncbi.nlm.nih.gov/sra (SRR5634347SRR5634826, SRR5629781-SRR5630260, SRR5630261-SRR5630452); genotypic data of the panel were obtained from She \& Jarosz (2018). Phenotypic data of $1125 \mathrm{~F}_{6}$ segregants for the mapping experiments are included in the Additional file 3. Phenotypic data of the main and the supplementary figures are available in the Additional file 4 and 5, respectively. All custom codes and a detailed documentation of the mapping of the IMA1-MAL locus are freely available on GitHub (https://github.com/peaceway33/PWH-Ferme ntationP2G). Correspondence and requests for materials and data should be addressed to Kevin Verstrepen. (kevin.verstrepen@kuleuven.be).

\section{Declarations}

Ethics approval and consent to participate Not applicable.

\section{Consent for publication}

Not applicable.

\section{Competing interests}

The authors declare no competing interests.

\section{Author details}

${ }^{1}$ VIB-KU Leuven Center for Microbiology, Leuven, Belgium. ${ }^{2}$ CMPG Laboratory of Genetics and Genomics, Department M2S, KU Leuven, Leuven, Belgium. ${ }^{3}$ Leuven Institute for Beer Research, Leuven, Belgium. ${ }^{4}$ Department of Chemical and Systems Biology, Stanford University School of Medicine, Stanford, CA 94305, USA. 5 Department of Developmental Biology, Stanford University School of Medicine, Stanford, CA 94305, USA. 'Labo VIB-CMPG, Bio-Incubator, Gaston Geenslaan 1, 3001 Leuven, Heverlee, Belgium. 
Received: 15 June 2021 Accepted: 16 October 2021

Published online: 02 November 2021

\section{References}

1. Gallone B, Steensels J, Prahl T, Soriaga L, Saels V, Herrera-Malaver B, et al. Domestication and divergence of saccharomyces cerevisiae beer yeasts. Cell. 2016;166(6):1397-1410.e16.

2. Steensels J, Gallone B, Voordeckers K, Verstrepen KJ. Domestication of industrial microbes. Curr Biol. 2019:29(10):R381-93.

3. Fay JC, Benavides JA. Evidence for domesticated and wild populations of Saccharomyces cerevisiae. PLoS Genet. 2005;1 (1):66-71.

4. Liti G, Carter DM, Moses AM, Warringer J, Parts L, James SA, et al. Population genomics of domestic and wild yeasts. Nature. 2009:458(7236):337-41.

5. Carreto L, Eiriz MF, Gomes AC, Pereira PM, Schuller D, Santos MAS. Comparative genomics of wild type yeast strains unveils important genome diversity. BMC Genomics. 2008;9:1-17.

6. Gallone B, Steensels J, Mertens S, Dzialo MC, Gordon JL, Wauters R, et al. Interspecific hybridization facilitates niche adaptation in beer yeast. Nat Ecol Evol. 2019:3(11):1562-75.

7. Steensels J, Verstrepen KJ. Taming wild yeast: Potential of conventional and nonconventional yeasts in industrial fermentations. Annu Rev Microbiol. 2014;68:61-80

8. Steensels J, Snoek T, Meersman E, Nicolino MP, Voordeckers K, Verstrepen $\mathrm{KJ}$. Improving industrial yeast strains: exploiting natural and artificial diversity. FEMS Microbiol Rev. 2014;38(5):947-95.

9. Meersman E, Steensels J, Paulus T, Struyf N, Saels V, Mathawan M, et al. Breeding strategy to generate robust yeast starter cultures for cocoa pulp fermentations. Appl Environ Microbiol. 2015:81(18):6166-76.

10. Sharon E, Chen S-AA, Khosla NM, Smith JD, Pritchard JK, Fraser HB. Functional genetic variants revealed by massively parallel precise genome editing. Cell. 2018;175(2):544-57.

11. Sadhu MJ, Bloom JS, Day L, Kruglyak L. CRISPR-directed mitotic recombination enables genetic mapping without crosses. Science (New York, NY) 2016;352(6289):1113-6.

12. Roy KR, Smith JD, Vonesch SC, Lin G, Tu CS, Lederer AR, et al. Multiplexed precision genome editing with trackable genomic barcodes in yeast. Nat Biotechnol. 2018:36(6):512-20.

13. Peltier E, Friedrich A, Schacherer J, Marullo P. Quantitative trait nucleotides impacting the technological performances of industrial saccharomyces cerevisiae strains. Front Genet. 2019;10:6

14. Long A, Liti G, Luptak A, Tenaillon O. Elucidating the molecular architecture of adaptation via evolve and resequence experiments. Nat Rev Genet. 2015:16(10):567-82.

15. Mans R, Daran JMG, Pronk JT. Under pressure: evolutionary engineering of yeast strains for improved performance in fuels and chemicals production. Curr Opin Biotechnol. 2018;50:47-56.

16. Sandberg TE, Salazar MJ, Weng LL, Palsson BO, Feist AM. The emergence of adaptive laboratory evolution as an efficient tool for biological discovery and industrial biotechnology. Metab Eng. 2019;56(August):1-16.

17. Voordeckers K, Kominek J, Das A, Espinosa-Cantú A, De Maeyer D, Arslan $A$, et al. Adaptation to high ethanol reveals complex evolutionary pathways. PLoS Genet. 2015;11(11):1-31.

18. Ho PW, Swinnen S, Duitama J, Nevoigt E. The sole introduction of two single-point mutations establishes glycerol utilization in Saccharomyces cerevisiae CENPK derivatives. Biotechnol Biofuels. 2017;10(1):1-15.

19. Huang CJ, Lu MY, Chang YW, Li WH. Experimental evolution of yeast for high-temperature tolerance. Mol Biol Evol. 2018;35(8):1823-39.

20. Brem RB, Kruglyak $L$. The landscape of genetic complexity across 5,700 gene expression traits in yeast. Proc Natl Acad Sci USA. 2005;102(5):1572-7.

21. Rockman MV, Kruglyak L. Genetics of global gene expression. Nat Rev Genet. 2006;7(11):862-72.

22. Steinmetz LM, Sinha H, Richards DR, Spiegelman JI, Oefner PJ, McCusker $\mathrm{JH}$, et al. Dissecting the architecture of a quantitative trait locus in yeast. Nature. 2002:416(6878):326-30.

23. Liti G, Louis EJ. Advances in quantitative trait analysis in yeast. PLoS Genet. 2012;8:8
24. Collard BCY, Jahufer MZZ, Brouwer JB, Pang ECK. An introduction to markers, quantitative trait loci (QTL) mapping and marker-assisted selection for crop improvement: The basic concepts. Euphytica. 2005:142(1-2):169-96.

25. Duitama J, Sánchez-Rodríguez A, Goovaerts A, Pulido-Tamayo S, Hubmann G, Foulquié-Moreno MR, et al. Improved linkage analysis of Quantitative Trait Loci using bulk segregants unveils a novel determinant of high ethanol tolerance in yeast. BMC Genomics. 2014;15:1.

26. Parts L, Cubillos FA, Warringer J, Jain K, Salinas F, Bumpstead SJ, et al. Revealing the genetic structure of a trait by sequencing a population under selection. Genome Res. 2011;21(7):1131-8.

27. Meijnen JP, Randazzo P, Foulquié-Moreno MR, Van Den Brink J, Vandecruys P, Stojiljkovic M, et al. Polygenic analysis and targeted improvement of the complex trait of high acetic acid tolerance in the yeast Saccharomyces cerevisiae. Biotechnol Biofuels. 2016;9(1):1-18.

28. Stojiljkovic M, Foulquié-Moreno MR, Thevelein JM. Polygenic analysis of very high acetic acid tolerance in the yeast Saccharomyces cerevisiae reveals a complex genetic background and several new causative alleles. Biotechnol Biofuels. 2020;13(1):1-27.

29. Hu XH, Wang MH, Tan T, Li JR, Yang H, Leach L, et al. Genetic dissection of ethanol tolerance in the budding yeast Saccharomyces cerevisiae. Genetics. 2007;175(3):1479-87.

30. Swinnen S, Schaerlaekens K, Pais T, Claesen J, Hubmann G, Yang Y, et al. Identification of novel causative genes determining the complex trait of high ethanol tolerance in yeast using pooled-segregant whole-genome sequence analysis. Genome Res. 2012;22(5):975-84.

31. Eder M, Sanchez I, Brice C, Camarasa C, Legras JL, Dequin S. QTL mapping of volatile compound production in Saccharomyces cerevisiae during alcoholic fermentation. BMC Genomics. 2018;19(1):1-19.

32. Bloom JS, Ehrenreich IM, Loo WT, Lite TLV, Kruglyak L. Finding the sources of missing heritability in a yeast cross. Nature. 2013;494(7436):234-7.

33. Rockman MV. The QTN program and the alleles that matter for evolution: All that's gold does not glitter. Evolution. 2012;66(1):1-17.

34. She R, Jarosz DF. Mapping Causal Variants with Single-Nucleotide Resolution Reveals Biochemical Drivers of Phenotypic Change. Cell. 2018:172(3):478-490.e15.

35. Jakobson CM, Jarosz DF. Molecular origins of complex heritability in natural genotype-to-phenotype relationships. Cell Syst. 2019;8(5):363-79.

36. Jakobson CM, She R, Jarosz DF. Pervasive function and evidence for selection across standing genetic variation in S cerevisiae. Nat Commun. 2019:10(1):1-13.

37. Peter J, De Chiara M, Friedrich A, Yue J-X, Pflieger D, Bergström A, et al. Genome evolution across 1,011 Saccharomyces cerevisiae isolates Species-wide genetic and phenotypic diversity. Nature. 2018;5:8.

38. Fournier T, Saada OA, Hou J, Peter J, Caudal E, Schacherer J. Extensive impact of low-frequency variants on the phenotypic landscape at population-scale. Life. 2019:8:1-18.

39. Skelly DA, Magwene PM. Population perspectives on functional genomic variation in yeast. Brief Funct Genomics. 2016;15(2):138-46.

40. McDonald J, Kreitman M. Adaptive protein evolution at Adh in Drosophila. Nature. 1991;351(June):652-4.

41. Mitchell-Olds T, Willis JH, Goldstein DB. Which evolutionary processes influence natural genetic variation for phenotypic traits? Nat Rev Genet. 2007:8(11):845-56.

42. Almeida P, Barbosa R, Zalar P, Imanishi Y, Shimizu K, Turchetti B, et al. A population genomics insight into the Mediterranean origins of wine yeast domestication. Mol Ecol. 2015;24(21):5412-27.

43. Weber C, Farwick A, Benisch F, Brat D, Dietz H, Subtil T, et al. Trends and challenges in the microbial production of lignocellulosic bioalcohol fuels. Appl Microbiol Biotechnol. 2010;87(4):1303-15.

44. Mohandesi N, Haghbeen K, Ranaei O, Arab SS, Hassani S. Catalytic efficiency and thermostability improvement of Suc2 invertase through rational site-directed mutagenesis. Enzyme Microb Technol. 2017:96:14-22.

45. Teste MA, Marie François J, Parrou JL. Characterization of a new multigene family encoding isomaltases in the yeast Saccharomyces cerevisiae, the IMA family. J Biol Chem. 2010;285(35):26815-24.

46. Voordeckers K, Brown CA, Vanneste K, van der Zande E, Voet A, Maere S, et al. Reconstruction of ancestral metabolic enzymes reveals molecular mechanisms underlying evolutionary innovation through gene duplication. PLoS Biol. 2012;10:12. 
47. Novak S, Zechner-Krpan V, Mari V. Maltose Transport and Metabolism in S cerevisiae. Ftb. 2004;42(3):213-8.

48. Offei B, Vandecruys P, De Graeve S, Foulquié-Moreno MR, Thevelein JM. Unique genetic basis of the distinct antibiotic potency of high acetic acid production in the probiotic yeast Saccharomyces cerevisiae var. Boulardii Genome Research. 2019;29(9):1478-94.

49. Umezu K, Amaya T, Yoshimoto A, Tomita K. Purification and properties of orotidine-5'-phosphate pyrophosphorylase and orotidine-5'-phosphate decarboxylase from bakers'yeast. J Biochem. 1971;70(2):249-62.

50. Alam MT, Zelezniak A, Mülleder M, Shliaha P, Schwarz R, Capuano F, et al. The metabolic background is a global player in Saccharomyces gene expression epistasis. Nat Microbiol. 2016;1(3):1-10.

51. Niederberger P, Miozzari G, Hütter R. Biological role of the general control of amino acid biosynthesis in Saccharomyces cerevisiae. Mol Cell Biol. 1981;1 (7):584-93.

52. Pais TM, Foulquié-Moreno MR, Hubmann G, Duitama J, Swinnen S, Goovaerts A, et al. Comparative Polygenic Analysis of Maximal Ethanol Accumulation Capacity and Tolerance to High Ethanol Levels of Cell Proliferation in Yeast. PLoS Genet. 2013;9:6.

53. Kohlhaw GB. Leucine Biosynthesis in Fungi: Entering Metabolism through the Back Door. Microbiol Mol Biol Rev. 2003;67(1):1-15.

54. Hazelwood LA, Daran JM, Van Maris AJA, Pronk JT, Dickinson JR. The Ehrlich pathway for fusel alcohol production: A century of research on Saccharomyces cerevisiae metabolism. Appl Environ Microbiol. 2008;74(8):2259-66.

55. Erdiwansyah E, Mamat R, Sani MSM, Khoerunnisa F, Sardjono RE, Ali O, et al. Effects of Diesel-Biodiesel Blends in Diesel Engine Single Cylinder on the Emission Characteristic. MATEC Web of Conferences. 2018;225:1-6.

56. Dzialo MC, Park R, Steensels J, Lievens B, Verstrepen KJ. Physiology, ecology and industrial applications of aroma formation in yeast. FEMS Microbiol Rev. 2017:41(2):S95-128.

57. Verstrepen KJ, Van Laere SDM, Vanderhaegen BMP, Derdelinckx G, Dufour JP, Pretorius IS, et al. Expression levels of the yeast alcohol acetyltransferase genes ATF1, Lg-ATF1, and ATF2 control the formation of a broad range of volatile esters. Appl Environ Microbiol. 2003;69(9):5228-37.

58. Goold HD, Kroukamp H, Williams TC, Paulsen IT, Varela C, Pretorius IS Yeast's balancing act between ethanol and glycerol production in lowalcohol wines. Microb Biotechnol. 2017:10(2):264-78.

59. Erasmus DJ, Cliff M, van Vuuren HJJ. Impact of yeast strain on the production of acetic acid, glycerol, and the sensory attributes of icewine. Am J Enol Vitic. 2004;55(4):371-8.

60. Remize F, Roustan IL, Sablayrolles JM, Barre P. Dequin S. Glycerol overproduction by engineered Saccharomyces cerevisiae wine yeast strains leads to substantial changes in by-product formation and to a stimulation of fermentation rate in stationary phase. Appl Environ Microbiol. 1999:65(1):143-9.

61. Jespersen L, Cesar LB, Meaden PG. Multiple-Glucoside Transporter Genes in Brewer's. Yeast. 1999;65(2):450-6.

62. Brown CA, Murray AW, Verstrepen KJ. Rapid expansion and functional divergence of subtelomeric gene families in yeasts. Curr Biol. 2010;20(10):895-903.

63. Naumoff DG, Naumov GI. Discovery of a novel family of a-glucosidase IMA genes in yeast Saccharomyces cerevisiae. Dokl Biochem Biophys. 2010;432(1):114-6.

64. Marques WL, Mans R, Marella ER, Cordeiro RL, van den Broek M, Daran $J M G$, et al. Elimination of sucrose transport and hydrolysis in Saccharomyces cerevisiae: A platform strain for engineering sucrose metabolism. FEMS Yeast Res. 2017:17(1):1-11.
65. He Y, Dong J, Yin $\mathrm{H}$, Zhao Y, Chen R, Wan X, et al. Wort composition and its impact on the flavour-active higher alcohol and ester formation of beer A review. J Inst Brew. 2014:120(3):157-63.

66. Younis OS, Stewart GG. Sugar uptake and subsequent ester and higher alcohol production by Saccharomyces cerevisiae. J Inst Brew. 1998;104(5):255-64.

67. Alexandre $\mathrm{H}$, Charpentier C. Biochemical aspects of stuck and sluggish fermentation in grape must. J Ind Microbiol Biotechnol. 1998;20(1):20-7.

68. Su Y, Zhang W, Zhang A, Shao W. Biorefinery: The production of isobutanol from biomass feedstocks. Applied Sciences (Switzerland). 2020;10(22):1-18.

69. Wess J, Brinek M, Boles E. Improving isobutanol production with the yeast Saccharomyces cerevisiae by successively blocking competing metabolic pathways as well as ethanol and glycerol formation. Biotechnol Biofuels. 2019;12(1):1-15.

70. Kurtz JE, Exinger F, Erbs P, Jund R. New insights into the pyrimidine salvage pathway of Saccharomyces cerevisiae: Requirement of six genes for cytidine metabolism. Curr Genet. 1999;36(3):130-6.

71. Hoffman CS, Winston F. A ten-minute DNA preparation from yeast efficiently releases autonomous plasmids for transformation of Escherichia coli. Gene. 1987;57(2-3):267-72.

72. Gietz RD, Schiestl RH. High-efficiency yeast transformation using the LiAc/SS carrier DNA/PEG method. Nat Protoc. 2007;2(1):31-4.

73. Steensels J, Meersman E, Snoek T, Saels V, Verstrepen KJ. Large-scale selection and breeding to generate industrial yeasts with superior aroma production. Appl Environ Microbiol. 2014;80(22):6965-75.

74. Wagih $O$. Parts $L$ gitter: a robust and accurate method for quantification of colony sizes from plate images. GBethesda. 2014;2:4

75. Bolger AM, Lohse M, Usadel B. Trimmomatic: A flexible trimmer for Illumina sequence data. Bioinformatics. 2014;30(15):2114-20.

76. Li H, Durbin R. Fast and accurate short read alignment with BurrowsWheeler transform. Bioinformatics. 2009;25(14):1754-60.

77. Poplin R, Ruano-Rubio V, DePristo M, Fennell T, Carneiro M, Van der Auwera $\mathrm{G}$, et al. Scaling accurate genetic variant discovery to tens of thousands of samples. Biorxiv. 2017:201:178.

78. Quinlan AR, Clark RA, Sokolova S, Leibowitz ML, Zhang Y, Hurles ME, et al. Genome-wide mapping and assembly of structural variant breakpoints in the mouse genome. Genome Res. 2010;20(5):623-35.

79. Thorvaldsdóttir H, Robinson JT, Mesirov JP. Integrative Genomics Viewer (IGV): High-performance genomics data visualization and exploration. Brief Bioinform. 2013;14(2):178-92.

80. Cingolani P, Platts A, Wang LL, Coon M, Nguyen T, Wang L, et al. A program for annotating and predicting the effects of single nucleotide polymorphisms, SnpEff: SNPs in the genome of Drosophila melanogaster strain w1118; iso-2; iso-3. Fly. 2012;6(2):80-92.

81. Szklarczyk D, Franceschini A, Wyder S, Forslund K, Heller D, Huerta-Cepas J, et al. STRING v10: Protein-protein interaction networks, integrated over the tree of life. Nucleic Acids Res. 2015:43(D1):D447-52.

82. Shannon P, Markiel A, Ozier O, Baliga NS, Wang JT, Ramage D, et al. Cytoscape: a software environment for integrated models of biomolecular interaction networks. Genome Res. 2003:13(11):2498-504.

\section{Publisher's Note}

Springer Nature remains neutral with regard to jurisdictional claims in published maps and institutional affiliations. 